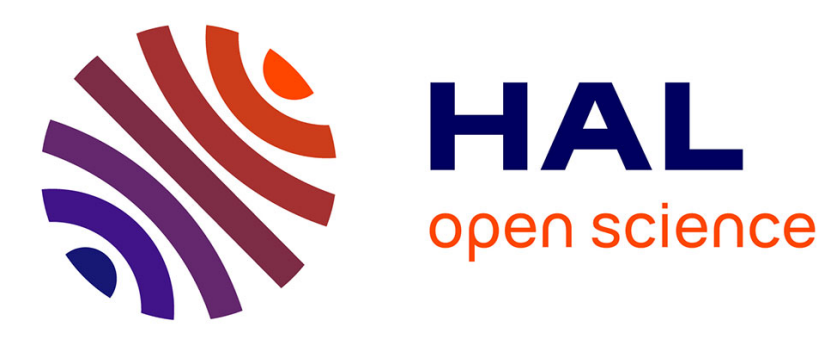

\title{
Multi-dimensional shear shallow water flows: problems and solutions
}

\author{
Sergey L. Gavrilyuk, Kseniya A. Ivanova, Nicolas Favrie
}

\section{To cite this version:}

Sergey L. Gavrilyuk, Kseniya A. Ivanova, Nicolas Favrie. Multi-dimensional shear shallow water flows: problems and solutions. Journal of Computational Physics, In press. hal-01529497

\section{HAL Id: hal-01529497 \\ https://hal.science/hal-01529497}

Submitted on 30 May 2017

HAL is a multi-disciplinary open access archive for the deposit and dissemination of scientific research documents, whether they are published or not. The documents may come from teaching and research institutions in France or abroad, or from public or private research centers.
L'archive ouverte pluridisciplinaire HAL, est destinée au dépôt et à la diffusion de documents scientifiques de niveau recherche, publiés ou non, émanant des établissements d'enseignement et de recherche français ou étrangers, des laboratoires publics ou privés. 


\title{
Multi-dimensional shear shallow water flows : problems and solutions
}

\author{
S. Gavrilyuk, K. Ivanovał, N. Favrie ${ }^{\ddagger}$ \\ May 25, 2017
}

\begin{abstract}
The mathematical model of shear shallow water flows of uniform density is studied. This is a $2 \mathrm{D}$ hyperbolic non-conservative system of equations which is reminiscent of a generic Reynoldsaveraged model of barotropic turbulent flows. The model has three families of characteristics corresponding to the propagation of surface waves, shear waves and average flow (contact characteristics). The system is non-conservative : for six unknowns (the fluid depth, two components of the depth averaged horizontal velocity, and three independent components of the symmetric Reynolds stress tensor) one has only five conservation laws (conservation of mass, momentum, energy and mathematical 'entropy'). A splitting procedure for solving such a system is proposed allowing us to define a weak solution. Each split subsystem contains only one family of waves (either surface or shear waves) and contact characteristics. The accuracy of such an approach is tested on exact 2D solutions describing the flows where the velocity is linear with respect to the space variables, and 1D solutions. The capacity of the model to describe the full transition observed in the formation of roll waves : from uniform flow to one-dimensional roll waves, and, finally, to 2D transverse 'fingering' of roll wave profiles is shown.
\end{abstract}

Key words : Non-conservative hyperbolic equations, Godunov-type methods, roll waves

\section{Introduction}

The Saint-Venant equations are of great importance both in hydraulic and oceanographic applications. The reason is that they are simpler compared to the $n$-dimensional Euler equations with a free surface. Indeed, the Saint-Venant equations describe the evolution of the fluid depth and depth averaged velocities defined in a fixed $n-1$ dimensional domain. They form a hyperbolic system of equations in conservative form for which standard numerical methods can be applied [22, 28, 48]. The derivation of Saint-Venant equations is based on the smallness of the parameter $\varepsilon=H / L$ where $H$ and $L$ are the vertical and horizontal scale lengths, respectively, and the hypothesis that the dependence of the horizontal velocity on the vertical coordinate is very weak, i. e. the fluid flow is almost potential (the flow is not sheared). Since the shear effects are completely neglected in the model derivation, the Saint-Venant equations are not able to describe neither the formation of large scale eddies ('roller') appearing in the hydraulic jumps near the free surface nor the form of the

\footnotetext{
${ }^{*}$ Corresponding author : Aix-Marseille Université, CNRS, IUSTI, UMR 7343, 5 rue E. Fermi, 13453 Marseille Cedex 13, France and Novosibirsk State University, 2 Pirogova street, 630090 Novosibirsk, Russia, sergey.gavrilyuk@univ-amu.fr

${ }^{\dagger}$ Aix-Marseille Université, CNRS, IUSTI, UMR 7343, 5 rue E. Fermi, 13453 Marseille Cedex 13, France, ivanova.kseniya15@gmail.com

¥Aix-Marseille Université, CNRS, IUSTI, UMR 7343, 5 rue E. Fermi, 13453 Marseille Cedex 13, France, nicolas.favrie@univ-amu.fr
} 
hydraulic jump. A natural extension of the Saint-Venant equations are integro-differential Benney's equations [6] describing shear shallow water flows of uniform density. They represent an infinite dimensional system of quasilinear equations, where the matrices are replaced by linear operators acting in some functional spaces. The notion of hyperbolicity of such an 'infinite-dimensional' system can also be introduced [44, 45, 29, 8]. Benney's equations admit, in particular, a linear vertical shear with constant vorticity as an exact solution. A numerical approach based on such a linear approximation was developed in [46] in $1 \mathrm{D}$ case. To the best of our knowledge, 2D computations of the Benney equations are absent in the literature.

For shear flows with varying in space and time vorticity an intermediate model was recently proposed where the governing equations are obtained by depth avergaing of Euler equations without assuming potential flow $[47,37,38,39]$. The hypothesis of smallness of the horizontal vorticity (the hypothesis of weakly sheared flows) allows us to keep the second order depth averaged correlations in the governing equations but neglect the third order correlations, and thus to close the governing system. This approach was further extended to the case where the third-order correlations are taken into account [13].

This intermediate multi-dimensional model is reminiscent of the classical Reynolds averaged Euler equations for the compressible barotropic turbulent flows [21]. The model complemented by friction terms was used for the study of 1D travelling waves down inclined plane (roll waves) and hydraulic jumps. A strong physical adequacy of the model with the experimental observations was found $[37,38,39]$.

The multi-dimensional case is much more challenging. For the flows over a flat bottom without friction effects, the system can be written in the form [47, 39, 20]:

$$
\begin{gathered}
h_{t}+\operatorname{div}(h \mathbf{u})=0, \\
(h \mathbf{u})_{t}+\operatorname{div}\left(h \mathbf{u} \otimes \mathbf{u}+\frac{g h^{2}}{2} \mathbf{I}+h \mathbf{P}\right)=\mathbf{0}, \\
\frac{D \mathbf{P}}{D t}+\frac{\partial \mathbf{u}}{\partial \mathbf{x}} \mathbf{P}+\mathbf{P}\left(\frac{\partial \mathbf{u}}{\partial \mathbf{x}}\right)^{T}=0, \frac{D}{D t}=\frac{\partial}{\partial t}+\mathbf{u} \cdot \nabla .
\end{gathered}
$$

Here $t$ is the time, $\mathbf{x}=(x, y)^{T}$ are the Cartesian coordinates, $\mathbf{u}=(u, v)^{T}$ is the depth averaged horizontal velocity, $h$ is the fluid depth, $g$ is the gravity, and $\mathbf{P}$ is the stress tensor which measures the distortion of the instantaneous horizontal velocity profile $\tilde{\mathbf{u}}(t, x, y, z)$ depending of the vertical coordinate $z$. The sign $\otimes$ means the tensor product, and $\mathbf{I}$ is the identity tensor. The definition of the depth averaged horizontal velocity $\mathbf{u}(t, x)$ and shear stress tensor $\mathbf{P}$ is as follows :

$$
\mathbf{u}(t, x)=\frac{1}{h} \int_{0}^{h} \tilde{\mathbf{u}}(t, x, y, z) d z, \mathbf{P}=\frac{1}{h} \int_{0}^{h}(\tilde{\mathbf{u}}-\mathbf{u}) \otimes(\tilde{\mathbf{u}}-\mathbf{u}) d z .
$$

The tensor $\mathbf{P}$ is symmetric and positive definite. The positive definiteness of $\mathbf{P}$ is a consequence of the Cauchy-Schwarz inequality.

The striking mathematical analogy with the Reynolds averaging equations of barotropic compressible turbulent flows $[31,51,52,20]$ allows us to call $\mathbf{R}=h \mathbf{P}$ the Reynolds stress tensor, and $\mathbf{P}$ the reduced Reynolds stress tensor. For mathematical reasons, the choice of $\mathbf{P}$ is more convenient than that of $\mathbf{R}$. For simplicity, both $\mathbf{R}$ and $\mathbf{P}$ will be further refereed to as the Reynolds stress tensor. ${ }^{1}$

\footnotetext{
${ }^{1}$ The evolution of $\mathbf{P}$ (the last equation of (1)) is not governed by any specific objective derivative, i.e. the equation is not invariant under the change of variables $t^{\prime}=t, \mathbf{x}^{\prime}=\mathbf{O}(t) \mathbf{x}, \mathbf{u}^{\prime}=\mathbf{O}(t) \mathbf{u}+\mathbf{\mathbf { O }} \mathbf{x}, \mathbf{P}^{\prime}=\mathbf{O P O} \mathbf{O}^{T}$. Here $\mathbf{O}(t)$ is a time-dependent orthogonal transformation : $\mathbf{O O}^{T}=\mathbf{I}$, ' $\mathrm{T}$ ' means transposition, and 'dot' denotes the time derivative. This is due to the fact that the tensor equation for the Reynolds stress tensor is not a geometric equation, but a physical one, representing a sort of micro-Newton law derived from the Euler equations by depth averaging. Thus, this equation should be only Galilean invariant, which is obviously the case.
} 
Equations (1) admit the energy conservation law:

$$
\frac{\partial}{\partial t}\left(h\left(\frac{1}{2}|\mathbf{u}|^{2}+e_{i}+e_{T}\right)\right)+\operatorname{div}\left(h \mathbf{u}\left(\frac{1}{2}|\mathbf{u}|^{2}+e_{i}+e_{T}\right)+\left(\frac{g h^{2}}{2} \mathbf{I}+h \mathbf{P}\right) \mathbf{u}\right)=0,
$$

where

$$
e_{T}=\frac{1}{2} \operatorname{tr} \mathbf{P}, \quad e_{i}=\frac{1}{2} g h
$$

and the conservation of 'entropy':

$$
\frac{\partial h \Psi}{\partial t}+\operatorname{div}(h \mathbf{u} \Psi)=0, \Psi=\frac{\operatorname{Det}(\mathbf{P})}{h^{2}} .
$$

The variable $\Psi$ is refereed to as 'entropy' because this quantity is transported along the mean flow as the true entropy for the Euler equations of compressible fluids. Also, we will see that this quantity will increase through the shocks in analogy with the conventional entropy (see $[37,38,39]$ for the 1 D study of roll waves and hydraulic jumps).

The system is hyperbolic but not in conservative form. The hyperbolicity was established, for example, in [7], for the equations of compressible turbulent flows, generalizing the system (1). However, the fact of the non-conservativity was just a 'feeling' which was not rigorously proved in the literature. In Appendix A we have established that the system (1) admits only five conservation laws written above : conservation of mass, momentum, energy and entropy. Since the number of scalar unknowns is six $(h, \mathbf{u}$ and three independent components of $\mathbf{P})$, the system is not in conservative form. The definition and computation of discontinuous solutions for non-conservative hyperbolic equations is a challenging problem (see examples of non-conservative systems in compressible turbulence [7, 2], multi-layer shallow water flows $[34,4,32,5,1,30]$, multi-phase fluid flows [3, 24, 40, 41, 42, 19, 14], solid-fluid systems [17, 33]).

Essentially, four approaches are used for numerical solving of non-conservative systems of equations. The most classical one is based on the Volpert path definition for non-conservative products [15]. The second one is the formulation of an augmented system of 'Rankine-Hugoniot relations' through the study of travelling wave solutions of an extended system of equations approximating a given system (formulation of kinetic relations) [50, 26]. The third one is based on the relaxation technique where the studied system is approximated by a new hyperbolic system with all linearly degenerate in the sense of Lax eigenfields $[9,14]$. Finally, the additional relations can be formulated from the compatibility between theoretical and experimental results [16, 42]. Excepting the first approach (more formal and hence less precise), all the approaches mentioned above are not universal : they are usually specific to the model under study.

In [18], we proposed a new splitting approach to the equations of hyperelasticity. This system in $3 \mathrm{D}$ case has three types of sonic waves (one longitudinal and two transverse) and contact characteristics. The system was split into several subsystems each of which contained only one type of sonic waves (only longitudinal or transverse). Each subsystem was hyperbolic and admitted a weak formulation. Such an approach was extended to non-conservative multi-solid systems [33]. It allowed us not only to define the non-conservative products, but also to increase the precision and robustness of the numerical method. Such a philosophy will also be developed here.

In section 2, the hyperbolicity of the system (1) is established. The Rankine -Hugioniot relations compatible to the positive definiteness of the Reynolds stress tensor are proposed in section 3. Dissipation terms are introduced in section 4. The splitting procedure and its numerical realisation are presented in sections $5,6,7$. The numerical results are shown in section 8 . Technical details are in Appendices A and B. 


\section{Hyperbolicity study}

The hyperbolicity study is analogous to that given in [7] for the compressible turbulent flows. For the sake of completeness, we present here a specific case of shear shallow water flows. Denoting the components of $\mathbf{P}$ by $P_{i j}, i, j=1,2$, one can rewrite the system (1) in Cartesian coordinates in the form :

$$
\begin{gathered}
h_{t}+u h_{x}+v h_{y}+h u_{x}+h v_{y}=0, \\
u_{t}+u u_{x}+v u_{y}+g h_{x}+\frac{1}{h}\left(h P_{11}\right)_{x}+\frac{1}{h}\left(h P_{12}\right)_{y}=0, \\
v_{t}+u v_{x}+v v_{y}+g h_{y}+\frac{1}{h}\left(h P_{12}\right)_{x}+\frac{1}{h}\left(h P_{22}\right)_{y}=0, \\
P_{11 t}+u P_{11 x}+v P_{11 y}+2 P_{11} u_{x}+2 P_{12} u_{y}=0, \\
P_{12 t}+u P_{12 x}+v P_{12 y}+P_{12}\left(u_{x}+v_{y}\right)+P_{11} v_{x}+P_{22} u_{y}=0, \\
P_{22 t}+u P_{22 x}+v P_{22 y}+2 P_{12} v_{x}+2 P_{22} v_{y}=0 .
\end{gathered}
$$

Or, in matrix form :

$$
\frac{\partial \mathbf{W}}{\partial t}+\mathbf{A} \frac{\partial \mathbf{W}}{\partial x}+\mathbf{B} \frac{\partial \mathbf{W}}{\partial y}=\mathbf{0}
$$

where

$$
\mathbf{W}=\left(\begin{array}{c}
h \\
u \\
v \\
P_{11} \\
P_{12} \\
P_{22}
\end{array}\right), \mathbf{A}=\left(\begin{array}{ccccccc}
u & h & 0 & 0 & 0 & 0 \\
\frac{g h+P_{11}}{h} & u & 0 & 1 & 0 & 0 \\
\frac{P_{12}}{h} & 0 & u & 0 & 1 & 0 \\
0 & 2 P_{11} & 0 & u & 0 & 0 \\
0 & P_{12} & P_{11} & 0 & u & 0 \\
0 & 0 & 2 P_{12} & 0 & 0 & u
\end{array}\right),
$$

The characteristic surfaces $S(t, x, y)=0$ for (4) satisfy the relations :

$$
\operatorname{det}\left(\mathbf{I} \frac{\partial S}{\partial t}+\mathbf{A} \frac{\partial S}{\partial x}+\mathbf{B} \frac{\partial S}{\partial y}\right)=0
$$


It implies :

$$
\begin{gathered}
\chi=0, \\
\chi= \pm \sqrt{(\nabla S)^{T} \mathbf{P} \nabla S}, \\
\chi= \pm \sqrt{g h|\nabla S|^{2}+3(\nabla S)^{T} \mathbf{P} \nabla S}
\end{gathered}
$$

where

$$
\chi=\frac{\partial S}{\partial t}+\mathbf{u} \cdot \nabla S, \nabla S=\left(\frac{\partial S}{\partial x}, \frac{\partial S}{\partial y}\right)^{T} .
$$

The characteristic field (7) is double, while the fields (8) and (9) are simple. They are real since $\mathbf{P}$ is positive definite. One can prove that the double field has two left linearly independent eigenvectors of the corresponding characteristic matrix. Hence, the equations are hyperbolic. To understand the structure of the eigenfields, consider the governing equations in $x$-direction. The eigenvalues of the matrix $\mathbf{A}$ are :

$$
\lambda_{1,2}=u, \quad \lambda_{3,4}=u \pm b, b=\sqrt{P_{11}}, \quad \lambda_{5,6}=u \pm a, a=\sqrt{g h+3 P_{11}} .
$$

For the multiple eigenvalue $\lambda_{1,2}=u$ we have two linearly independent right eigenvectors :

$$
\begin{array}{cc}
\mathbf{r}_{1}=(0,0,0,0,0,1)^{T}, & \nabla_{\mathbf{W}} \lambda_{1} \cdot \mathbf{r}_{1}=0, \\
\mathbf{r}_{2}=\left(-h, 0,0, g h+P_{11}, P_{12}, 0\right)^{T}, & \nabla_{\mathbf{W}} \lambda_{2} \cdot \mathbf{r}_{2}=0,
\end{array}
$$

For the eigenvalue $\lambda_{3}=u+b$ one has:

$$
\mathbf{r}_{3}=\left(0,0, b, 0, b^{2}, 2 P_{12}\right)^{T}, \quad \nabla_{\mathbf{W}} \lambda_{3} \cdot \mathbf{r}_{3}=0,
$$

For the eigenvalue $\lambda_{4}=u-b$ one has:

$$
\mathbf{r}_{4}=\left(0,0,-b, 0, b^{2}, 2 P_{12}\right)^{T}, \quad \nabla_{\mathbf{W}} \lambda_{4} \cdot \mathbf{r}_{4}=0
$$

For the eigenvalue $\lambda_{5}=u+a$ one has:

$$
\begin{gathered}
\mathbf{r}_{5}=\left\{h, a, \frac{2 a P_{12}}{a^{2}-b^{2}}, 2 b^{2}, \frac{a^{2}+b^{2}}{a^{2}-b^{2}} P_{12}, \frac{4 P_{12}^{2}}{a^{2}-b^{2}}\right\}^{T}, \\
\nabla_{\mathbf{W}} \lambda_{5} \cdot \mathbf{r}_{5}=\frac{3}{2 a}\left(a^{2}+b^{2}\right)>0,
\end{gathered}
$$

For the eigenvalue $\lambda_{6}=u-a$ one has:

$$
\begin{gathered}
\mathbf{r}_{6}=\left\{h,-a,-\frac{2 a P_{12}}{a^{2}-b^{2}}, 2 b^{2}, \frac{a^{2}+b^{2}}{a^{2}-b^{2}} P_{12}, \frac{4 P_{12}^{2}}{a^{2}-b^{2}}\right\}^{T}, \\
\nabla_{\mathbf{W}} \lambda_{6} \cdot \mathbf{r}_{6}=-\frac{3}{2 a}\left(a^{2}+b^{2}\right)<0 .
\end{gathered}
$$

The fields corresponding to the multiple eigenvalues $\lambda_{1,2}=u$ and to $\lambda_{3,4}=u \pm b$ are linear degenerate in the sense of Lax, while the fields $\lambda_{5,6}=u \pm a$ are genuinely non-linear. The family $\lambda_{3,4}=u \pm b$ (further on referred to as $b$-waves) is reminiscent of the shear waves in hyperelasticity, while the family $\lambda_{5,6}=u \pm a$ (further on referred to as $a$-waves) is reminiscent of the longitudinal waves. The analogy with shear waves in hyperelasticity and $b$-waves was also noticed in [49] in incompressible limit. An interesting analogy between the linearised equations of incompressible turbulence and Maxwell's equations was also underlined there. 


\section{Rankine-Hugoniot relations}

Since the number of scalar conservation laws is only five (mass, momentum, energy and 'entropy'), while the number of unknowns is six ( $h, \mathbf{u}$ and three independent components of $\mathbf{P})$, the system is not in conservative form. The proof is given in Appendix A.

For discontinuous solutions, the Rankine-Hugoniot relations coming from the mass, momentum and energy equations are :

$$
\begin{gathered}
{\left[h\left(\mathbf{u} \cdot \mathbf{n}-D_{n}\right)\right]=0} \\
{\left[h \mathbf{u}\left(\mathbf{u} \cdot \mathbf{n}-D_{n}\right)+\frac{g h^{2}}{2} \mathbf{n}+h \mathbf{P n}\right]=0,} \\
{\left[h\left(\mathbf{u} \cdot \mathbf{n}-D_{n}\right)\left(\frac{1}{2}|\mathbf{u}|^{2}+e_{i}+e_{T}\right)+\mathbf{n}^{T}\left(\frac{g h^{2}}{2} \mathbf{I}+h \mathbf{P}\right) \mathbf{u}\right]=0 .}
\end{gathered}
$$

Here for any function $f$ we denote $[f]=f^{+}-f^{-}$, where $f^{+}, f^{-}$are the right and the left limit values of $f$ at the discontinuity surface, $D_{n}$ is the normal velocity of the surface, and $\mathbf{n}$ is the normal unit vector to the surface. We denote also by $\mathbf{s}$ the tangent unit vector to the surface such that $(\mathbf{n}, \mathbf{s})$ form a Cartesian basis. The momentum equation implies:

$$
\begin{gathered}
{\left[h\left(\mathbf{u} \cdot \mathbf{n}-D_{n}\right)^{2}+\frac{g h^{2}}{2}+h \mathbf{n}^{T} P \mathbf{n}\right]=0,} \\
{\left[h\left(\mathbf{u} \cdot \mathbf{n}-D_{n}\right)(\mathbf{u} \cdot \mathbf{s})+h \mathbf{s}^{T} P \mathbf{n}\right]=0 .}
\end{gathered}
$$

As usually, we distinguish two types of discontinuities: contact discontinuities (interfaces) where $\mathbf{u} \cdot \mathbf{n}-D_{n}=0$, and shock waves where $\mathbf{u} \cdot \mathbf{n}-D_{n} \neq 0$.

\subsection{Contact discontinuities}

We consider first the interfaces $\mathbf{u} \cdot \mathbf{n}-D_{n}=0$. We obtain two relations following from the momentum equation :

$$
\begin{gathered}
{\left[\frac{g h^{2}}{2}+h \mathbf{n}^{T} \mathbf{P n}\right]=0,} \\
{\left[\mathbf{s}^{T} h \mathbf{P n}\right]=0,}
\end{gathered}
$$

and the energy equation:

$$
\left[(\mathbf{u} \cdot \mathbf{s}) \mathbf{s}^{T} h \mathbf{P n}\right]=0 .
$$

So, we need to distinguish two types of contact discontinuities. The first type is determined by the condition that at each side of the contact discontinuity the tangential component of the stress vector $\mathbf{s}^{T} h \mathbf{P n}$ vanishes. Then, a priori, the jump of the tangential velocity can be arbitrary:

$$
[\mathbf{u} \cdot \mathbf{s}] \neq 0
$$

So, the sliding is admitted as in the case of contact discontinuities for the Euler equations of compressible fluids.

For the second type of contact discontinuity where the tangential component of the stress vector $\mathbf{s}^{T} h \mathbf{P n}$ is continuous, but not necessarily vanishing, the sliding is forbidden :

$$
[\mathbf{u} \cdot \mathbf{s}]=0 .
$$

So, the full velocity vector should be continuous. This kind of interfaces is not admitted by the Euler equations of compressible fluids. 


\subsection{Shocks}

We consider the discontinuity interfaces where $\mathbf{u} \cdot \mathbf{n}-D_{n} \neq 0$. They are called shocks. The RankineHugoniot relations coming from the mass, momentum (two scalar relations) and energy equations are :

$$
\begin{gathered}
{\left[h\left(\mathbf{u} \cdot \mathbf{n}-D_{n}\right)\right]=0,} \\
{\left[h\left(\mathbf{u} \cdot \mathbf{n}-D_{n}\right)^{2}+\frac{g h^{2}}{2}+h \mathbf{n}^{T} P \mathbf{n}\right]=0, \quad\left[h\left(\mathbf{u} \cdot \mathbf{n}-D_{n}\right)(\mathbf{u} \cdot \mathbf{s})+h \mathbf{s}^{T} P \mathbf{n}\right]=0,} \\
{\left[h\left(\mathbf{u} \cdot \mathbf{n}-D_{n}\right)\left(\frac{1}{2}|\mathbf{u}|^{2}+e_{i}+e_{T}\right)+(\mathbf{u} \cdot \mathbf{n})\left(\frac{g h^{2}}{2}+h \mathbf{n}^{T} \mathbf{P n}\right)+(\mathbf{u} \cdot \mathbf{s}) h \mathbf{s}^{T} \mathbf{P n}\right]=0 .}
\end{gathered}
$$

Contrary to the Euler equations of compressible fluids, the momentum equation allows also a sliding along the shocks surfaces. This system of Rankine-Hugoniot relations is obviously not sufficient to describe the full structure of strong discontinuities. As it is proved in Appendix A all linearly independent conservation laws admitted by the system (1) of the form :

$$
\frac{\partial f}{\partial t}+\frac{\partial g_{1}}{\partial x}+\frac{\partial g_{2}}{\partial y}=0
$$

are only those of mass, momentum, energy and $h \Psi$. Here $f, g_{1}, g_{2}$ are functions depending on $h$, $\mathbf{u}$ and $\mathbf{P}$. Thus, there is no hope to define a 'classical' weak solution to (1). Moreover, the shock relation coming from the equation for

$$
\Psi=\frac{\operatorname{Det}(\mathbf{P})}{h^{2}} .
$$

implies the continuity of $\Psi$ through the shocks :

$$
[\Psi]=0 .
$$

However, this condition is not physically acceptable. Indeed, for 1D flows the system of RankineHugoniot relations is closed. Moreover, the 1D system admits an analogue of $\Psi$ having a clear physical meaning. This quantity is associated with the enstrophy (squared vorticity) and plays the role of 'mathematical entropy' : it increases through the shock. For example, for hydraulic jumps the enstrophy increase corresponds to the vorticity creation : a roller appears at the forward slope of the hydraulic jump [37, 38]. Also, in [19] a simplified model of multi-phase flows was proposed, and a quantity caracterising the micro-energy of interaction between phases was introduced, that is analogous to $\Psi$. This quantity was also increasing through the shock.

The non-conservative nature of the multi-dimensional equations of shear shallow water flows represents an enormous difficulty from the mathematical and numerical point of view. Moreover, when the shocks appear, we also should be aware about positive definiteness of $\mathbf{P}$ : this property should be guaranteed on the weak solutions. If the solution is smooth, this property is easy to establish. Indeed, the equation for $\Psi$ can be integrated in the Lagrangian coordinates $\mathbf{X}$ related to the mean flow. One can write in the Lagrangian coordinates :

$$
\Psi(t, \mathbf{X})=\Psi(0, \mathbf{X}),
$$

or

$$
\frac{\lambda_{1} \lambda_{2}}{h^{2}}=\frac{\lambda_{10} \lambda_{20}}{h_{0}^{2}}
$$

where $\lambda_{i}, i=1,2$ are eigenvalues of $\mathbf{P}$, and the index 0 corresponds to the initial state. It is then clear that if $\mathbf{P}$ is initially positive, it will be positive for any time, if the solution is smooth.

We will establish below a set of additional Rankine-Hugoniot relations which guarantee the positive definiteness of $\mathbf{P}$ even. Also, dissipative terms compatible with the positive definiteness of $\mathbf{P}$ will also be introduced into the governing equations. 


\subsubsection{Positive definiteness of the Reynolds stress through the shock : $1 \mathrm{D}$ case study}

This case was already studied in $[47,37,38]$. Here, we present it for completeness. The governing equations are :

$$
\begin{gathered}
\frac{\partial h}{\partial t}+\frac{\partial h u}{\partial x}=0 \\
\frac{\partial h u}{\partial t}+\frac{\partial}{\partial x}\left(h u^{2}+\frac{g h^{2}}{2}+\Phi h^{3}\right)=0 \\
\frac{\partial}{\partial t}\left(\frac{h}{2}\left(u^{2}+g h+\Phi h^{2}\right)\right)+\frac{\partial}{\partial x}\left(h u\left(\frac{u^{2}}{2}+g h+\frac{3}{2} \Phi h^{2}\right)\right)=0 .
\end{gathered}
$$

Here $\Phi=P_{11} / h^{2}$ is the enstrophy which conserves along trajectories :

$$
\frac{D \Phi}{D t}=0
$$

The variable $\Phi$ is a one-dimensional analogue of $\Psi$ ( $P_{11}$ is is a 'one-dimensional' determinant of P.) The governing equations (18)-(20) are reminiscent of the Euler equations of compressible fluids with the specific internal energy given by

$$
e=\frac{g h}{2}+\frac{\Phi h^{2}}{2}
$$

and the pressure :

$$
p=\frac{g h^{2}}{2}+\Phi h^{3}
$$

Finally, the Rankine-Hugoniot relations should be augmented by the inequality : the enstrophy $\Phi$ increases through the shock. This is also equivalent the fluid depth increase. This is reminiscent of standard Rankine-Hugoniot relations for the Euler equations of compressible fluids. Thus, $P_{11}$ stays positive.

\subsubsection{Positive definiteness of the Reynolds stress through the shock : 2D case study}

The previous $1 \mathrm{D}$ example suggests us a hypothesis about the following set of Rankine-Hugoniot relations. Let us suppose that

$$
\begin{gathered}
{\left[\frac{\mathbf{s}^{T} P \mathbf{n}}{h}\right]=0,} \\
{\left[\mathbf{s}^{T} P \mathbf{s}\right]=0 .}
\end{gathered}
$$

Then the ' entropy' $\Psi$ of the system is increasing through the shock if and only if the 'normal' entropy $\frac{\mathbf{n}^{T} P \mathbf{n}}{h^{2}}$ is increasing through the shock. Indeed, this fact follows from the identity :

$$
\begin{gathered}
\Psi=\frac{\left(\mathbf{n}^{T} P \mathbf{n}\right)\left(\mathbf{s}^{T} P \mathbf{s}\right)-\left(\mathbf{s}^{T} P \mathbf{n}\right)^{2}}{h^{2}}=\left(\frac{\mathbf{n}^{T} P \mathbf{n}}{h^{2}}\right)\left(\mathbf{s}^{T} P \mathbf{s}\right)-\left(\frac{\left(h \mathbf{s}^{T} P \mathbf{n}\right)^{2}}{h^{4}}\right) \\
=\left(\frac{\mathbf{n}^{T} P \mathbf{n}}{h^{2}}\right)\left(\mathbf{s}^{T} P \mathbf{s}\right)_{0}-\left(\frac{\left(h \mathbf{s}^{T} P \mathbf{n}\right)^{2}}{h^{4}}\right)_{0}>\left(\frac{\mathbf{n}^{T} P \mathbf{n}}{h^{2}}\right)_{0}\left(\mathbf{s}^{T} P \mathbf{s}\right)_{0}-\left(\frac{\left(h \mathbf{s}^{T} P \mathbf{n}\right)^{2}}{h^{4}}\right)_{0}=\Psi_{0},
\end{gathered}
$$

where the index ' 0 ' denotes the state before the shock. The justification of the jump relations (24), (25) which guarantees the inequality (26) will be done below by using a specific splitting procedure in solving the non-conservative equations. 


\section{Dissipative terms compatible with the positive definite- ness of the Reynolds stress tensor}

We add now dissipation effects in the model :

$$
\begin{gathered}
h_{t}+\operatorname{div}(h \mathbf{u})=0 \\
(h \mathbf{u})_{t}+\operatorname{div}\left(h \mathbf{u} \otimes \mathbf{u}+\frac{g h^{2}}{2} \mathbf{I}+h \mathbf{P}\right)=-C_{f}|\mathbf{u}| \mathbf{u}, \\
\frac{D \mathbf{P}}{D t}+\frac{\partial \mathbf{u}}{\partial \mathbf{x}} \mathbf{P}+\mathbf{P}\left(\frac{\partial \mathbf{u}}{\partial \mathbf{x}}\right)^{T}=\mathcal{D}, \quad \mathcal{D}=\mathcal{D}^{T}
\end{gathered}
$$

Here $C_{f}$ is the Chézy coefficient, and $\mathbf{D}$ is the dissipation tensor to be defined. The equations $(27)$ should also satisfy the energy conservation law :

$$
\frac{\partial}{\partial t}\left(h\left(\frac{1}{2}|\mathbf{u}|^{2}+e_{i}+e_{T}\right)\right)+\operatorname{div}\left(h \mathbf{u}\left(\frac{1}{2}|\mathbf{u}|^{2}+e_{i}+e_{T}\right)+\left(\frac{g h^{2}}{2} \mathbf{I}+h \mathbf{P}\right) \mathbf{u}\right)=-C_{f}|\mathbf{u}|^{3}-Q
$$

where the dissipative source term $Q$ should be positive. The positivity of $Q$ is an analogue of the second low of thermodynamics. The compatibility of (27) and (28) implies the expression for $Q$ in terms of $\mathcal{D}$ :

$$
\operatorname{tr}(\mathcal{D})=-\frac{2}{h} Q
$$

The equations for the reduced stress tensor $\mathbf{P}$ can be written in Cartesian coordinates as follows :

$$
\begin{gathered}
\frac{D P_{11}}{D t}+2 P_{11} u_{x}+2 P_{12} u_{y}=\mathcal{D}_{11}, \\
\frac{D P_{12}}{D t}+P_{12}\left(u_{x}+v_{y}\right)+P_{11} v_{x}+P_{22} u_{y}=\mathcal{D}_{12} \\
\frac{D P_{22}}{D t}+2 P_{12} v_{x}+2 P_{22} v_{y}=\mathcal{D}_{22} .
\end{gathered}
$$

It implies the evolution equation for $\Psi$ :

$$
h^{2} \frac{D}{D t}\left(\frac{P_{11} P_{22}-P_{12}^{2}}{h^{2}}\right)=\mathcal{D}_{11} P_{22}-2 P_{12} \mathcal{D}_{12}+\mathcal{D}_{22} P_{11}
$$

Or, in an invariant form,

$$
h^{2} \frac{D}{D t}\left(\frac{\operatorname{det} \mathbf{P}}{h^{2}}\right)=\operatorname{tr}(\mathbf{P}) \operatorname{tr}(\mathcal{D})-\operatorname{tr}(\mathbf{P} \mathcal{D})
$$

By analogy with the Stokes hypotheses we assume that the dissipation tensor $\mathcal{D}$ is an isotropic tensor function of $\mathbf{P}$. Then, in two-dimensional case, $\mathcal{D}$ is linear in $\mathbf{P}$ :

$$
\mathcal{D}=-\frac{2}{h}|\mathbf{u}|^{3}\left(\alpha \mathbf{P}+\frac{\delta}{2} \mathbf{I}\right)
$$

where $\alpha$ and $\delta$ are functions of invariants of $\mathbf{P}$. The multiplier $-2|\mathbf{u}|^{3} / h$ is for convenience only. So, $\alpha$ has the dimension $s^{2} m^{-2}$, while $\delta$ is dimensionless. Consider the simplest case where $\delta=0$. This choice allows us the reduction to the Saint-Venant equations in the limit $\mathbf{P}=\mathbf{0}$. One has then :

$$
\mathcal{D}=-\frac{2 \alpha}{h}|\mathbf{u}|^{3} \mathbf{P}
$$


In particular, this implies the equation for $\Psi$ in the form :

$$
h^{2} \frac{D}{D t}\left(\frac{\operatorname{det}(\mathbf{P})}{h^{2}}\right)=-\frac{4 \alpha}{h}|\mathbf{u}|^{3} \operatorname{det}(\mathbf{P}) .
$$

Then (29) and (30) imply the following relation between $Q$ and $\alpha$ :

$$
Q=\alpha \operatorname{tr}(\mathbf{P})|\mathbf{u}|^{3} .
$$

We will finally choose $Q$ as in [38] :

$$
\alpha \operatorname{tr}(\mathbf{P})=\max \left(0, C_{r} \frac{\frac{\operatorname{tr}(\mathbf{P})}{h^{2}}-\varphi}{\frac{\operatorname{tr}(\mathbf{P})}{h^{2}}}\right) .
$$

Here $\varphi$ and $C_{r}$ are the model constants. $\varphi$ is associated to the enstrophy of small vortexes at the vicinity of the bottom, and $C_{r}$ is a dissipation coefficient associated to the roller formation $[37,38,39]$. The coefficients $\varphi$ and $C_{r}$ were evaluated there from experimental data. We will indicate these specific values below. The formula (33) allows us to recover 1D case studied in [38]. As it follows from (31) and (33), the 'entropy' $\Psi$ is decreasing on continuous solutions, but always stays positive. This means that the dissipation law also conserves the positive definiteness of $\mathbf{P}$.

A simplified option for the dissipative term could also be as follows :

$$
\alpha \operatorname{tr}(\mathbf{P})=C_{r}
$$

Then

$$
Q=C_{r}|\mathbf{u}|^{3}
$$

and

$$
\mathcal{D}=-\frac{2}{h} C_{r}|\mathbf{u}|^{3} \frac{\mathbf{P}}{\operatorname{tr}(\mathbf{P})}
$$

Such a choice also guarantees the positive definiteness of $\mathbf{P}$ and thus is reasonable from the physical point of view. However, the wave profile obtained with such a law does not correspond to the experimental ones. Indeed, Brock $[10,11,12]$ measured the stationary roll wave profiles in different conditions (different slopes and wall roughness). He noticed that the roll wave profiles contain always the following three essential parts: first, a sudden increasing of the depth since all waves break i.e. acquire steeply sloping wave front, second, a continuous zone where the depth increases progressively, and third, a slowly decreasing zone until a new hydraulic jump (see such a profile in Figure 6). This is a reason to prefer the option (33) because it allows us to reproduce experimental profiles.

\section{$5 \quad$ Splitting method in Cartesian coordinates}

For convenience, we write here once again the governing equations (1) in Cartesian coordinates (4):

$$
\begin{gathered}
h_{t}+u h_{x}+v h_{y}+h u_{x}+h v_{y}=0 \\
u_{t}+u u_{x}+v u_{y}+g h_{x}+\frac{1}{h}\left(h P_{11}\right)_{x}+\frac{1}{h}\left(h P_{12}\right)_{y}=0, \\
v_{t}+u v_{x}+v v_{y}+g h_{y}+\frac{1}{h}\left(h P_{12}\right)_{x}+\frac{1}{h}\left(h P_{22}\right)_{y}=0, \\
P_{11 t}+u P_{11 x}+v P_{11 y}+2 P_{11} u_{x}+2 P_{12} u_{y}=0, \\
P_{12 t}+u P_{12 x}+v P_{12 y}+P_{12}\left(u_{x}+v_{y}\right)+P_{11} v_{x}+P_{22} u_{y}=0,
\end{gathered}
$$




$$
P_{22 t}+u P_{22 x}+v P_{22 y}+2 P_{12} v_{x}+2 P_{22} v_{y}=0 .
$$

As mentioned earlier, the system is hyperbolic but not in conservative form. We will define now an almost conservative formulation of the governing equations based on a splitting procedure allowing us to define the discontinuous solutions. A conventional geometric splitting of (4) is first applied consisting in solving of the governing equations first in $x$ and then in $y$ direction. We will do two steps more for each uni-directional subsystem referring to this as a 'physical' splitting. Roughly, speaking, each uni-directional subsystem, describing two types of waves (surface and shear waves), is split into two subsystems which are hyperbolic and contains only one type of waves ( $a$ or $b$ waves). Each physical subsystem admits its own energy conservation law, and its own 'entropy'. In physical terms, one can say that one performs a thermodynamically compatible splitting. An analogous splitting was proposed earlier in [18] in hyperelasticity.

Consider first the subsystem in $x$-direction :

$$
\begin{gathered}
h_{t}+u h_{x}+h u_{x}=0, \\
u_{t}+u u_{x}+g h_{x}+\frac{1}{h}\left(h P_{11}\right)_{x}=0, \\
v_{t}+u v_{x}+\frac{1}{h}\left(h P_{12}\right)_{x}=0, \\
P_{11 t}+u P_{11 x}+2 P_{11} u_{x}=0, \\
P_{12 t}+u P_{12 x}+P_{12} u_{x}+P_{11} v_{x}=0, \\
P_{22 t}+u P_{22 x}+2 P_{12} v_{x}=0 .
\end{gathered}
$$

As mentioned earlier, the system is hyperbolic and admits three types of waves : a contact discontinuity propagating with the velocity $u$, surface gravity waves propagating with the velocity $u \pm a$, and shear waves propagating with a smaller velocity $u \pm b$. The eigenfield corresponding to the contact discontinuity and the $b$-waves are linearly degenerate in the sense of Lax, while the eigenfield corresponding to $a$-waves is genuinely non-linear in the sense of Lax. The idea is to split (35) into two subsystems treating separately $a$-waves and $b$-waves. The subsystem for $a$-waves is:

$$
\begin{gathered}
h_{t}+u h_{x}+h u_{x}=0, \\
u_{t}+u u_{x}+g h_{x}+\frac{1}{h}\left(h P_{11}\right)_{x}=0, \\
v_{t}+u v_{x}=0, \\
P_{11 t}+u P_{11 x}+2 P_{11} u_{x}=0, \\
P_{12 t}+u P_{12 x}+P_{12} u_{x}=0, \\
P_{22 t}+u P_{22 x}=0 .
\end{gathered}
$$

The equations (36) admit the following conservative form :

$$
\begin{gathered}
h_{t}+(u h)_{x}=0, \\
(h u)_{t}+\left(h u^{2}+g \frac{h^{2}}{2}+h P_{11}\right)_{x}=0, \\
(h v)_{t}+(h u v)_{x}=0, \\
\left(h\left(\frac{u^{2}+v^{2}}{2}+\frac{g h}{2}+\frac{P_{11}+P_{22}}{2}\right)\right)_{t}+\left(h u\left(\frac{u^{2}+v^{2}}{2}+\frac{g h}{2}+\frac{P_{11}+P_{22}}{2}\right)+\frac{g h^{2}}{2} u+h P_{11} u\right)_{x}=0 . \\
P_{12 t}+\left(u P_{12}\right)_{x}=0,
\end{gathered}
$$




$$
\left(h P_{22}\right)_{t}+\left(h u P_{22}\right)_{x}=0 .
$$

They also admit the 'entropy' equation :

$$
\frac{D}{D t}\left(\frac{P_{11} P_{22}-P_{12}^{2}}{h^{2}}\right)=0, \frac{D}{D t}=\frac{\partial}{\partial t}+u \frac{\partial}{\partial x},
$$

an the 'enstrophy' equation :

$$
\frac{D}{D t}\left(\frac{P_{11}}{h^{2}}\right)=0
$$

One solves the 6 conservation laws (37) at this step. The 'entropy' will increase through the shock. Indeed, the ratio $P_{12} / h$ and the component $P_{22}$ are conserved through the shock, so we need only the increase of $P_{11} / h^{2}$ what is the case of 1 D flows [37, 38].

The subsystem for $b$-waves is:

$$
\begin{gathered}
h_{t}=0, \\
(h u)_{t}=0, \\
(h v)_{t}+\left(h P_{12}\right)_{x}=0, \\
P_{11 t}=0, \\
P_{12 t}+P_{11} v_{x}=0, \\
P_{22 t}+2 P_{12} v_{x}=0 .
\end{gathered}
$$

An 'almost' conservative form (38) for $b$-waves is :

$$
\begin{gathered}
h_{t}=0, \\
(h u)_{t}=0, \\
(h v)_{t}+\left(h P_{12}\right)_{x}=0, \\
P_{11 t}=0, \\
P_{12 t}+P_{11} v_{x}=0, \\
\left(h\left(\frac{v^{2}}{2}+\frac{P_{22}}{2}\right)\right)_{t}+\left(h P_{12} v\right)_{x}=0 .
\end{gathered}
$$

The shock relations for the equation for $P_{12}$ are well defined because in this subsystem $P_{11}$ is continuous through shocks. The 'entropy' conservation law :

$$
\frac{\partial}{\partial t}\left(\frac{P_{11} P_{22}-P_{12}^{2}}{h^{2}}\right)=0
$$

and the 'enstrophy' conservation law :

$$
\frac{\partial}{\partial t}\left(\frac{P_{11}}{h^{2}}\right)=0
$$

are consequences of (39). Since the characteristic field corresponding to $b$-waves is linearly degenerate, the velocity of the shocks is equal to the corresponding eigenvalue. As a consequence, the conservation of the energy is equivalent to the conservation of the 'entropy' through the shock. Thus, the positive definiteness of $\mathbf{P}$ is guaranteed even in the presence of shocks.

The study of the system in $y$-direction is analogous. Indeed, this system is :

$$
h_{t}+v h_{y}+h v_{y}=0,
$$




$$
\begin{gathered}
u_{t}+v u_{y}+\frac{1}{h}\left(h P_{12}\right)_{y}=0, \\
v_{t}+v v_{y}+g h_{y}+\frac{1}{h}\left(h P_{22}\right)_{y}=0, \\
P_{11 t}+v P_{11 y}+2 P_{12} u_{y}=0, \\
P_{12 t}+v P_{12 y}+P_{12} v_{y}+P_{22} u_{y}=0, \\
P_{22 t}+v P_{22 y}+2 P_{22} v_{y}=0 .
\end{gathered}
$$

The equations for $a$-waves and $b$-waves in $y$-direction are obtained from the corresponding equations (37) and (39) by the change of variables $u \rightarrow v, x \rightarrow y, P_{11} \rightarrow P_{22}, P_{22} \rightarrow P_{11}$.

\section{Mathematical properties of split systems}

\subsection{Subsystem 1 for $a$-waves}

The subsystem (37) can be rewritten in the following form:

$$
\frac{\partial \mathbf{W}}{\partial t}+\mathbf{A}(\mathbf{W}) \frac{\partial \mathbf{W}}{\partial x}=\mathbf{0}
$$

where the vector of unknowns $\mathbf{W}$ and matrix $\mathbf{A}$ are defined as:

$$
\begin{gathered}
\mathbf{W}=\left(h, u, v, P_{11}, P_{12}, P_{22}\right)^{T}, \\
\mathbf{A}=\left[\begin{array}{cccccc}
u & h & 0 & 0 & 0 & 0 \\
\left(g h+P_{11}\right) / h & u & 0 & 1 & 0 & 0 \\
0 & 0 & u & 0 & 0 & 0 \\
0 & 2 P_{11} & 0 & u & 0 & 0 \\
0 & P_{12} & 0 & 0 & u & 0 \\
0 & 0 & 0 & 0 & 0 & u
\end{array}\right] .
\end{gathered}
$$

The eigenvalues of $\mathbf{A}$ are :

$$
\lambda_{1,2,3,4}=u, \lambda_{5,6}=u \pm a, a=\sqrt{g h+3 P_{11}} .
$$

For the multiple eigenvalue $\lambda_{1,2,3,4}=u$ we have 4 linearly independent eigenvectors:

$$
\begin{gathered}
\mathbf{r}_{1}=\left(h, 0,0,-\left(g h+P_{11}\right), 0,0\right)^{T}, \nabla_{\mathbf{W}} \lambda_{1} \cdot \mathbf{r}_{1}=0 \\
\mathbf{r}_{2}=(0,0,1,0,0,0)^{T}, \nabla_{\mathbf{W}} \lambda_{2} \cdot \mathbf{r}_{2}=0 \\
\mathbf{r}_{3}=(0,0,0,0,1,0)^{T}, \nabla_{\mathbf{W}} \lambda_{3} \cdot \mathbf{r}_{3}=0 \\
\mathbf{r}_{4}=(0,0,0,0,0,1)^{T}, \nabla_{\mathbf{W}} \lambda_{4} \cdot \mathbf{r}_{4}=0
\end{gathered}
$$

For the eigenvalue $\lambda_{5}=u+a$ one has:

$$
\mathbf{r}_{5}=\left(h, a, 0,2 b^{2}, P_{12}, 0\right)^{T}, \quad \nabla_{\mathbf{w}} \lambda_{5} \cdot \mathbf{r}_{5}=\frac{3}{2 a}\left(a^{2}+b^{2}\right)>0 .
$$


For the eigenvalue $\lambda_{6}=u-a$ one has:

$$
\mathbf{r}_{6}=\left(h,-a, 0,2 P_{11}, P_{12}, 0\right)^{T}, \quad \nabla_{\mathbf{W}} \lambda_{6} \cdot \mathbf{r}_{6}=-\frac{3}{2 a}\left(a^{2}+b^{2}\right)<0 .
$$

The fields corresponding to $\lambda=u$ are linearly degenerate in the sense of Lax. For $\lambda=u \pm a$, the fields are genuinely nonlinear in the sense of Lax (compare this result with the general $1 \mathrm{D}$ case).

The eigenvectors are linearly independent. Indeed,

$$
\operatorname{det}\left[\mathbf{r}_{1}, \mathbf{r}_{2}, \mathbf{r}_{3}, \mathbf{r}_{4}, \mathbf{r}_{5}, \mathbf{r}_{6}\right]=-2 a^{3} h \neq 0 .
$$

Hence, the subsystem (37) or (41) is hyperbolic.

\subsection{Subsystem 2 for $b$-waves}

The subsystem (39) can also be rewritten in the form

$$
\frac{\partial \mathbf{W}}{\partial t}+\mathbf{A}(\mathbf{W}) \frac{\partial \mathbf{W}}{\partial x}=\mathbf{0}
$$

where the matrix $\mathbf{A}$ is

$$
\mathbf{A}=\left[\begin{array}{cccccc}
0 & 0 & 0 & 0 & 0 & 0 \\
0 & 0 & 0 & 0 & 0 & 0 \\
P_{12} / h & 0 & 0 & 0 & 1 & 0 \\
0 & 0 & 0 & 0 & 0 & 0 \\
0 & 0 & P_{11} & 0 & 0 & 0 \\
0 & 0 & 2 P_{12} & 0 & 0 & 0
\end{array}\right]
$$

It implies:

$$
\operatorname{det}(\mathbf{A}-\lambda \mathbf{I})=\lambda^{4}\left(P_{11}-\lambda^{2}\right)=0 .
$$

Hence, one has 6 real eigenvalues:

$$
\lambda_{1,2,3,4}=0, \lambda_{5,6}= \pm b, b=\sqrt{P_{11}} .
$$

For the multiple eigenvalue $\lambda_{1,2,3,4}=0$ one has 4 linearly independent eigenvectors:

$$
\begin{gathered}
\mathbf{r}_{1}=\left(1,0,0,0,-\frac{P_{12}}{h}, 0\right)^{T}, \quad \nabla_{\mathbf{W}} \lambda_{1} \cdot \mathbf{r}_{1}=0 \\
\mathbf{r}_{2}=(0,1,0,0,0,0)^{T}, \quad \nabla_{\mathbf{W}} \lambda_{2} \cdot \mathbf{r}_{2}=0, \\
\mathbf{r}_{3}=(0,0,0,1,0,0)^{T}, \quad \nabla_{\mathbf{W}} \lambda_{3} \cdot \mathbf{r}_{3}=0, \\
\mathbf{r}_{4}=(0,0,0,0,0,1)^{T}, \quad \nabla_{\mathbf{W}} \lambda_{4} \cdot \mathbf{r}_{4}=0
\end{gathered}
$$

For $\lambda_{5}=b$ one has :

$$
\mathbf{r}_{5}=\left(0,0, b, 0, b^{2}, 2 P_{12}\right), \quad \nabla_{\mathbf{w}} \lambda_{5} \cdot \mathbf{r}_{5}=0
$$

For $\lambda_{6}=-b$ one has :

$$
\mathbf{r}_{6}=\left(0,0,-b, 0, b^{2}, 2 P_{12}\right), \quad \nabla_{\mathbf{W}} \lambda_{6} \cdot \mathbf{r}_{6}=0 .
$$


The eigenvectors are linearly independent. Indeed:

$$
\operatorname{det}\left[\mathbf{r}_{1}, \mathbf{r}_{2}, \mathbf{r}_{3}, \mathbf{r}_{4}, \mathbf{r}_{5}, \mathbf{r}_{6}\right]=-2 P_{11} \sqrt{P_{11}} \neq 0 \text {, if } P_{11} \neq 0 \text {. }
$$

Hence, the subsystem (39) is hyperbolic. For this subsystem all eigenfields are linearly degenerate in the sense of Lax.

\section{$7 \quad$ Numerical scheme}

The numerical approach for (4) consists in solving first the model in $x$-direction, and then in $y$ direction. For each direction, the two subsystems for $a$ - and $b$-waves are solved successively. A Godunov-type scheme augmented by a correction step (see below) is used for this aim. Finally, the source terms are integrated. The vector of unknowns for all subsystems is :

$$
\mathbf{U}=\left[h, h u, h v, h P_{11}, P_{12}, h P_{22}, h E\right]^{T} .
$$

Here

$$
E=\left(u^{2}+v^{2}+g h+P_{11}+P_{22}\right) / 2 .
$$

\subsection{First subsystem : $a$-waves}

The first subsystem (called 'subsystem 1') augmented with the energy conservation law for $a$-waves is :

$$
\left\{\begin{array}{cc}
h_{t}+(u h)_{x}=0, & (a) \\
(h u)_{t}+\left(h u^{2}+p\right)_{x}=0, & (b) \\
(h v)_{t}+(h u v)_{x}=0, & (c) \\
\left(h P_{11}\right)_{t}+\left(h u P_{11}\right)_{x}+2 h P_{11} u_{x}=0, & (d) \\
P_{12 t}+\left(u P_{12}\right)_{x}=0, & (e) \\
\left(h P_{22}\right)_{t}+\left(h u P_{22}\right)_{x}=0 . & (f) \\
(h E)_{t}+(h E u+p u)_{x}=0, & (g)
\end{array}\right.
$$

with $p=g h^{2} / 2+h P_{11}$.

The equation (55.d) is not in conservative form, i.e. the product $P_{11} u_{x}$ is not well defined across discontinuities. Hopefully, this system is overdetermined and the correct value of $P_{11}$ will be obtained using the energy equation $(55 . \mathrm{g})$. This system is solved in 3 steps which can be summarized as follows:

- Solve the Riemann problem using any Riemann solver.

- Evolve all the conservative variables using a classical Godunov scheme.

- Compute $P_{11}$ from the energy equation

Each step is detailed hereafter. 


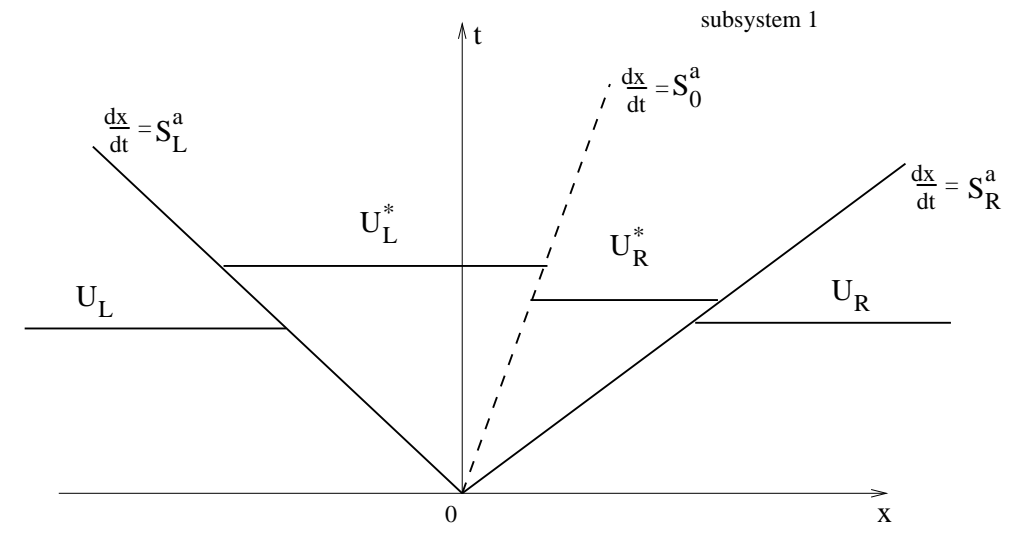

Figure 1: HLLC approximate Riemann solver for the subsystem 1 describing $a$-waves. Solution in the 'star' region consists of two constant states separated by a middle wave of speed $S_{0}^{a}=u^{*}$. The fastest and slowest waves denoted by $S_{L}^{a}$ and $S_{R}^{a}$, respectively, are estimated by using Davis' approximation.

\subsubsection{HLLC Riemann solver for subsystem 1}

For the first subsystem (55) the wave scheme is shown in Figure 1. The Rankine-Hugoniot relations read :

$$
\begin{gathered}
-[h] S^{a}+[h u]=0, \\
-[h u] S^{a}+\left[h u^{2}+p\right]=0, \\
-[h v] S^{a}+[h u v]=0, \\
-\left[P_{12}\right] S^{a}+\left[u P_{12}\right]=0, \\
-\left[h P_{22}\right] S^{a}+\left[h u P_{22}\right]=0, \\
-[h E] S^{a}+[h E u+p u]=0 .
\end{gathered}
$$

Here $S^{a}$ is the speed of the discontinuity, and $[f]$ means the jump of any variable $f$. The jump relation for the mass equations can be written in the following form :

$$
\left[h\left(u-S^{a}\right)\right]=0 \text {, i.e. } h\left(u-S^{a}\right)=m=\text { const } .
$$

Let us denote

$$
\begin{gathered}
m_{L}=h_{L}\left(u_{L}-S_{L}^{a}\right)=h_{L}^{*}\left(u^{*}-S_{L}^{a}\right) \\
m_{R}=h_{R}\left(u_{R}-S_{R}^{a}\right)=h_{R}^{*}\left(u^{*}-S_{R}^{a}\right) .
\end{gathered}
$$

Here the speeds of the left and right facing waves are obtained by using Davis' approximation :

$$
S_{R}^{a}=\max \left(u_{L}+a_{L}, u_{R}+a_{R}\right), S_{L}^{a}=\min \left(u_{L}-a_{L}, u_{R}-a_{R}\right),
$$

with $a_{L, R}^{2}=g h_{L, R}+3 P_{11 L, R}$. The Rankine-Hugoniot relations imply the continuity of the following parameters in the 'star regions':

$$
u_{L}^{*}=u_{R}^{*}=u^{*}, v_{L}^{*}=v_{L}, v_{R}^{*}=v_{R}, p_{L}^{*}=p_{R}^{*}=p^{*},
$$

with

$$
p=\frac{g h^{2}}{2}+h P_{11} .
$$


The momentum conservation law implies :

$$
u^{*}=\frac{p_{L}-p_{R}+m_{L} u_{L}-m_{R} u_{R}}{m_{L}-m_{R}},
$$

and

$$
p^{*}=\frac{m_{L} m_{R}\left(u_{R}-u_{L}\right)+m_{L} p_{R}-p_{L} m_{R}}{m_{L}-m_{R}} .
$$

Finally, the conservative variables in the 'star region' are:

$$
\begin{gathered}
h_{L, R}^{*}=m_{L, R} /\left(u^{*}-S_{L, R}^{a}\right), \\
h_{L, R}^{*} u_{L, R}^{*}=\left(h_{L, R} u_{L, R}\left(u_{L, R}-S_{L, R}^{a}\right)+p_{L, R}-p^{*}\right) /\left(u^{*}-S_{L, R}^{a}\right), \\
h_{L, R}^{*} v_{L, R}^{*}=h_{L, R} v_{L, R}\left(u_{L, R}-S_{L, R}^{a}\right) /\left(u^{*}-S_{L, R}^{a}\right), \\
P_{12 L, R}^{*}=P_{12 L, R}\left(u_{L, R}-S_{L, R}^{a}\right) /\left(u^{*}-S_{L, R}^{a}\right), \\
h_{l, r}^{*} E_{L, R}^{*}=\left(h_{L, R} E_{L, R}\left(u_{L, R}-S_{L, R}^{a}\right)+p_{L} u_{L}-p^{*} u^{*}\right) /\left(u^{*}-S_{L, R}^{a}\right) .
\end{gathered}
$$

These relations allow us to construct an approximate Riemann solver.

\subsubsection{Evolution with the Godunov type method}

The subsystem 1 can be rewritten in the following form :

$$
\frac{\partial \mathbf{U}}{\partial t}+\frac{\partial \mathbf{F}}{\partial x}=0 .
$$

Here the vector of conservative variables $\mathbf{U}$ and the vector of fluxes $\mathbf{F}$ are :

$$
\begin{gathered}
\mathbf{U}=\left(h, h u, h v, P_{12}, h P_{22}, h E\right)^{T}, \\
\mathbf{F}(\mathbf{U})=\left(h u, h u^{2}+p, h u v, u P_{12}, h u P_{22}, h u E+p u\right)^{T} .
\end{gathered}
$$

For simplicity, we use here the same generic notation $\mathbf{U}$ for the vector of conservative variables, even if this vector does not contain the component $h P_{11}$. Using the flux solution obtained in section 7.1.1 at the edge of each cells $\left(F^{*}\right)$, it is possible to evolve the conservative variables with the following scheme for the $i^{t h}$ cell:

$$
\mathbf{U}_{i}^{n+1}=\mathbf{U}_{i}^{n}-\frac{\Delta t}{\Delta x}\left(\mathbf{F}_{i+1 / 2}^{*, n}-\mathbf{F}_{i-1 / 2}^{*, n}\right) .
$$

Here $\Delta x$ is the discretisation step in the $x$-direction, $\Delta t$ is the time step verifiing the CourantFriedrichs-Lewy (CFL) condition $\left(\Delta t \leq \Delta x / S_{\max }\right)$. At this step the non-conservative equation for $h P_{11}$ is removed.

\subsubsection{Computation of $h P_{11}$}

Since the system is overdetermined, the non-conservative term $h P_{11}$ is obtained by using the total energy equation :

$$
h P_{11}=2 h E-g h^{2}-h P_{22}-\frac{(h u)^{2}+(h v)^{2}}{h} .
$$




\subsection{Subsystem $2: b$-waves}

The subsystem for $b$-waves (called 'subsystem 2'), augmented with the energy conservation law, reads:

$$
\left\{\begin{array}{cc}
h_{t}=0, & (a) \\
(h u)_{t}=0, & (b) \\
(h v)_{t}+\left(h P_{12}\right)_{x}=0, & (c) \\
\left(h P_{11}\right)_{t}=0, & (d) \\
P_{12 t}+P_{11} v_{x}=0, & (e) \\
\left(h P_{22}\right)_{t}+2 h P_{12} v_{x}=0 . & (f) \\
(h E)_{t}+\left(h P_{12} v\right)_{x}=0 . & (g)
\end{array}\right.
$$

Again, the system (59) is overdetermined. One can note that there are two nonconservative equations (59.e) and (59.f). The product $P_{11} v_{x}$ in (59.e) is well defined since $P_{11}$ is conserved through the shock. It is not the case for the term $2 h P_{12} v_{x}$ in equation (59.f). Since the jump relation is not well defined, there is no hope to obtain a good value of $P_{22}$. In the first step, this equation will be removed. Then, the energy conservation law will be again used to compute the value of $h P_{22}$. The numerical procedure will be similar to $a$-wave procedure :

- Solve the Riemann problem using any Riemann solver.

- Evolve all the conservative variables using a classical Godunov scheme.

- Compute $h P_{22}$ from the energy equation.

These steps and some important remarks on the evolution of the entropy will be detailed hereafter.

\subsubsection{HLLC Riemann solver for $b$-waves}

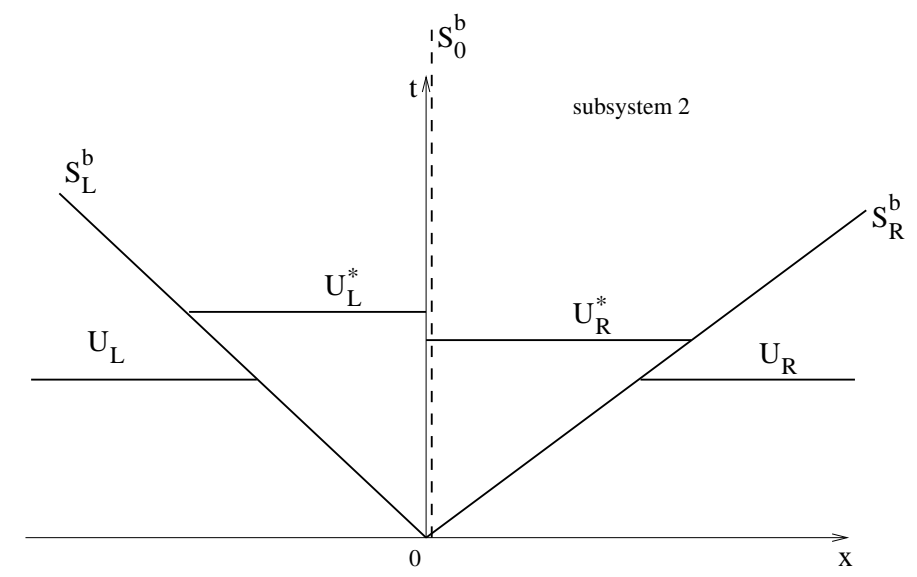

Figure 2: HLLC approximate Riemann solver for the subsystem 2 describing $b$-waves. Solution in the 'star' region consists of two constant states separated by a middle wave of speed $S_{0}^{b}=0$. The fastest and slowest waves $S_{R}^{b}$ and $S_{L}^{b}$, respectively, are estimated by using Davis' approximation.

The subsystem 2 for $b$-waves can be rewritten in the following form:

$$
\frac{\partial \mathbf{U}}{\partial t}+\frac{\partial \mathbf{F}}{\partial x}+\mathbf{K} \frac{\partial v}{\partial x}=0
$$

with

$$
\begin{gathered}
\mathbf{U}=\left(h, h u, h v, h P_{11}, P_{12}, h E\right)^{T}, \\
\mathbf{F}=\mathbf{F}(\mathbf{U})=\left(0,0, h P_{12}, 0,0, h P_{12} v\right)^{T}, \\
\mathbf{K}=\mathbf{K}(\mathbf{U})=\left(0,0,0,0, P_{11}, 0\right)^{T} .
\end{gathered}
$$


Again, for simplicity, the generic notation $\mathbf{U}$ for the 'conservative vector is used, even if this vector does not contain now the component $h P_{22}$. The wave scheme is shown in Figure 2. The set of jump relations for the subsystem 2 is :

$$
\begin{gathered}
-[h] S^{b}=0,-[u] S^{b}=0,-\left[P_{11}\right] S^{b}=0, \\
-[h v] S^{b}+\left[h P_{12}\right]=0, \\
-\left[h\left(\frac{v^{2}+P_{22}}{2}\right)\right] S^{b}+\left[h v P_{12}\right]=0,
\end{gathered}
$$

Through the contact discontinuity $\left(S^{b}=0\right)$ a priori one has :

$$
[h] \neq 0, \quad[u] \neq 0, \quad\left[P_{11}\right] \neq 0,
$$

but

$$
h_{L}^{*} P_{12 L}^{*}=h_{R}^{*} P_{12 R}^{*}=\left(h P_{12}\right)^{*}, \quad v^{*}=v_{L}^{*}=v_{R}^{*} .
$$

Through the shocks $\left(S^{b} \neq 0\right)$ one has from (62):

$$
h_{L}=h_{L}^{*}, \quad h_{R}=h_{R}^{*}, \quad u_{R}=u_{R}^{*}, \quad P_{11 R}=P_{11 R}^{*} .
$$

Equation (63) gives :

$$
\left(h P_{12}\right)^{*}=h_{R} h_{L} \frac{S_{R}^{b} P_{12 L}-S_{L}^{b} P_{12 R}-S_{L}^{b} S_{R}^{b}\left(v_{L}-v_{R}\right)}{h_{R} S_{R}^{b}-h_{L} S_{L}^{b}}
$$

and

$$
v^{*}=\frac{h_{L}\left(P_{12 L}-S_{L}^{b} v_{L}\right)-h_{R}\left(P_{12 R}-S_{R}^{b} v_{R}\right)}{h_{R} S_{R}^{b}-h_{L} S_{L}^{b}} .
$$

The extreme wave speeds can be estimated by Davis' approximation :

$$
S_{L}^{b}=\min \left(-\sqrt{P_{11 L}},-\sqrt{P_{11 R}}\right), \quad S_{R}^{b}=\max \left(\sqrt{P_{11 L}}, \sqrt{P_{11 R}}\right),
$$

Since the eigenfields is linearly degenerate, the wave speed on the right (left) only depend on the right (left) state. Thus, another possibility is to use

$$
S_{L}^{b}=-\sqrt{P_{11 L}}, \quad S_{R}^{b}=\sqrt{P_{11 R}} .
$$

This choice, more precise, is used for all the numerical results presented here. These relations allows us to construct an approximate Riemann solver.

\subsubsection{Godunov type scheme for subsystem 2}

The non-conservative equations for $P_{12}$ and $P_{22}$ necessitate a specific numerical treatment. For the equations in conservative form, we use the folowing Godunov type scheme :

$$
\mathbf{U}_{i}^{n+1}=\mathbf{U}_{i}^{n}+\frac{\Delta t}{\Delta x}\left(\mathbf{F}_{i+1 / 2}^{*, n}-\mathbf{F}_{i-1 / 2}^{*, n}\right)+\mathbf{K}_{i}^{n}\left(v_{i+1 / 2}^{*, n}-v_{i-1 / 2}^{*, n}\right) .
$$

\subsubsection{Computation of $h P_{22}$}

The non-conservative term $h P_{22}$ is obtained by using the total energy equation :

$$
h P_{22}=2 h E-g h^{2}-h P_{11}-\frac{(h u)^{2}+(h v)^{2}}{h} .
$$




\subsubsection{Conservation of the 'entropy'}

It is striking that the subsystem (59) conserves also the mathematical 'entropy'. Indeed, consider the Rankine-Hugoniot relations :

$$
\left\{\begin{array}{c}
-[v] S^{b}+\left[P_{12}\right]=0 \\
-\left[\left(v^{2}+P_{22}\right) / 2\right]+\left[P_{12} v\right] S^{b}=0
\end{array}\right.
$$

Here, for any $f,[f]=f-f_{0}$, where the index ' 0 ' means the state before the shock. It implies :

$$
\left\{\begin{array}{c}
P_{12}=\left(P_{12}\right)_{0}+S^{b}\left(v-v_{0}\right), \\
P_{22}=\left(P_{22}\right)_{0}+\left[2 P_{12} v\right] / S^{b}-\left[v^{2}\right],
\end{array}\right.
$$

We will show now that

$$
\operatorname{det} \mathbf{P}-\operatorname{det} \mathbf{P}_{0}=P_{11} P_{22}-P_{12}^{2}-\left(\left(P_{11}\right)_{0}\left(P_{22}\right)_{0}-\left(P_{12}\right)_{0}^{2}\right)
$$

vanishes through the shock. Since $h$ is continuous through the shock, it implies the conservation of the 'entropy'. As $P_{11}$ is continuous, we obtain :

$$
\operatorname{det} \mathbf{P}-\operatorname{det} \mathbf{P}_{0}=\left(P_{11}\right)_{0}\left(P_{22}-\left(P_{22}\right)_{0}\right)+\left(\left(P_{12}\right)_{0}-P_{12}\right)\left(\left(P_{12}\right)_{0}+P_{12}\right) .
$$

The equalities (72) imply :

$$
\begin{gathered}
\operatorname{det} \mathbf{P}-\operatorname{det} \mathbf{P}_{0}=\left(P_{11}\right)_{0}\left(\frac{\left[2 P_{12} v\right]}{S^{b}}-\left[v^{2}\right]\right)-S^{b}[v]\left(2\left(P_{12}\right)_{0}+S^{b}\left(v-v_{0}\right)\right)= \\
=\left(P_{11}\right)_{0}\left(2 \frac{\left(\left(P_{12}\right)_{0}+S^{b}\left(v-v_{0}\right)\right) v-\left(P_{12}\right)_{0} v_{0}}{S}-\left[v^{2}\right]\right)-S^{b}\left(v-v_{0}\right)\left(2\left(P_{12}\right)_{0}+S^{b}\left(v-v_{0}\right)\right)= \\
=\left(P_{11}\right)_{0}\left(2\left(v-v_{0}\right) v-\left[v^{2}\right]\right)-\left(S^{b}\right)^{2}\left(v-v_{0}\right)^{2} .
\end{gathered}
$$

As $\left(S^{b}\right)^{2}=P_{11}$, we obtain :

$$
\operatorname{det} \mathbf{P}-\operatorname{det} \mathbf{P}_{0}=\left(P_{11}\right)_{0}\left(2\left(v-v_{0}\right) v-\left(v^{2}-v_{0}^{2}\right)-\left(v-v_{0}\right)^{2}\right)=0 .
$$

The 'entropy' $\Psi=\frac{\operatorname{det} \mathbf{P}}{h^{2}}$ is thus conserved because the corresponding eigenfields are linearly degenerate in the sense of Lax. Finally, the entropy is increasing after solving the first subsystem, and does not change in solving the second subsystem.

\subsection{Integrating the source terms}

To add the source terms, we integrate the ordinary differential equations :

$$
\frac{d \mathbf{U}}{d t}=\mathbf{S}(\mathbf{U})
$$

with the full unknown vector $\mathbf{U}=\left(h, h u, h v, h P_{11}, P_{12}, h P_{22}, h E\right)^{T}$. The initial condition $\left.\mathbf{U}\right|_{t=0}=$ $\overline{\mathbf{U}}^{n+1}$ are obtained from the previous splitting steps. Here the vector of source terms is :

$$
\mathbf{S}(\mathbf{U})=\left(0,-g h \nabla b-C_{f} \mathbf{u}|\mathbf{u}|, h D_{11}, D_{12}, h D_{22},-g h \nabla b \cdot \mathbf{u}-C_{f}|\mathbf{u}|^{3}-Q\right)^{T},
$$


where

$$
|\mathbf{u}|=\sqrt{u^{2}+v^{2}}, D_{i j}=-\frac{2 \alpha}{h}|\mathbf{u}|^{3} P_{i j}, Q=\alpha \operatorname{tr}(\mathbf{P})|\mathbf{u}|^{3}, \alpha=\max \left(0, C_{r} \frac{\frac{\operatorname{tr}(\mathbf{P})}{h^{2}-\varphi}}{\frac{t^{2}(\mathbf{P})}{h^{2}}}\right), \nabla b=\left(\frac{\partial b}{\partial x}, \frac{\partial b}{\partial y}\right)^{T} .
$$

We added here the bottom topography $z=b(x, y)$. The equations are written in the reference frame where the gravity is orthogonal to the $(x, y)$-plane. So, the case of a mild slop bottom is considered. In applications, we will consider only the case of constant slope bottom. For example,

$$
-\nabla b=(\tan \theta, 0), \theta>0
$$

where $\theta$ is the inclination angle. Even if we have 7 equations for 6 variables, the equations are compatible : the energy equation is a consequence of the equations of mass, momentum and stress tensor.

This system of ordinary differential equation is solved by the first order scheme with the Euler method. A second order Runge-Kutta method is used for the higher order extension.

\subsection{Algorithm summary}

First, the 2D system is split into two 1D systems along each spatial direction. Then, each 1D system is successively split into two additional 1D subsystems. All the systems are hyperbolic. The numerical algorithm can be summarized as follows:

1. Subsystem 1 in $x$-direction.

- Solve the Riemann Problem in $x$-direction for the subsystem 1 at each cell boundary without source terms. The approximate HLLC solver was used for this aim.

- Evolve all flow variables with the Godunov-type method.

- Correction of the non-conservative variable $\left(h P_{11}\right)$ using the energy conservation law.

2. Subsystem 2 in $x$-direction.

- Solve the Riemann Problem in $x$-direction for the subsystem 2 at each cell boundary.

- Evolve for the conservative equations flow variables with the Godunov-type method.

- The correction of the non-conservative variable $\left(h P_{22}\right)$ using energy conservation law.

3. The same procedure is repeated in $y$-direction by changing $u \rightarrow v, x \rightarrow y, P_{11} \rightarrow P_{22}$, $P_{22} \rightarrow P_{11}$.

4. Integration of the source terms

5. Start again for the next time step.

\section{$8 \quad$ Numerical Results}

In this section, we will present numerical results obtained with the splitting procedure described above. 


\subsection{D shear test problem}

We solve here the governing equations in the case of vanishing source term. The initial discontinuity is located at $x=0.5 \mathrm{~m}$. The initial depth $h$ is $0.01 \mathrm{~m}$, the normal velocity $u$ is zero everywhere, the components of the stress tensor are $P_{11}=P_{22}=10^{-4} \mathrm{~m}^{2} / \mathrm{s}^{2}, P_{12}=0 \mathrm{~m}^{2} / \mathrm{s}^{2}$, the tangential velocity $v$ is $0.2 \mathrm{~m} / \mathrm{s}$ on the left, and $-0.2 \mathrm{~m} / \mathrm{s}$ on the right. Thus, the solution depends only on $x / t$. The first order Godunov method is used, with CFL number 0.3. The tangential velocity, tangential stress $P_{12}$ and stress $P_{22}$ are shown in Figure 3 at time instant $10 s$ for 500, 1000 and 10000 grid cells. The other variables do not evolve in time, they are shown in Figure 4.
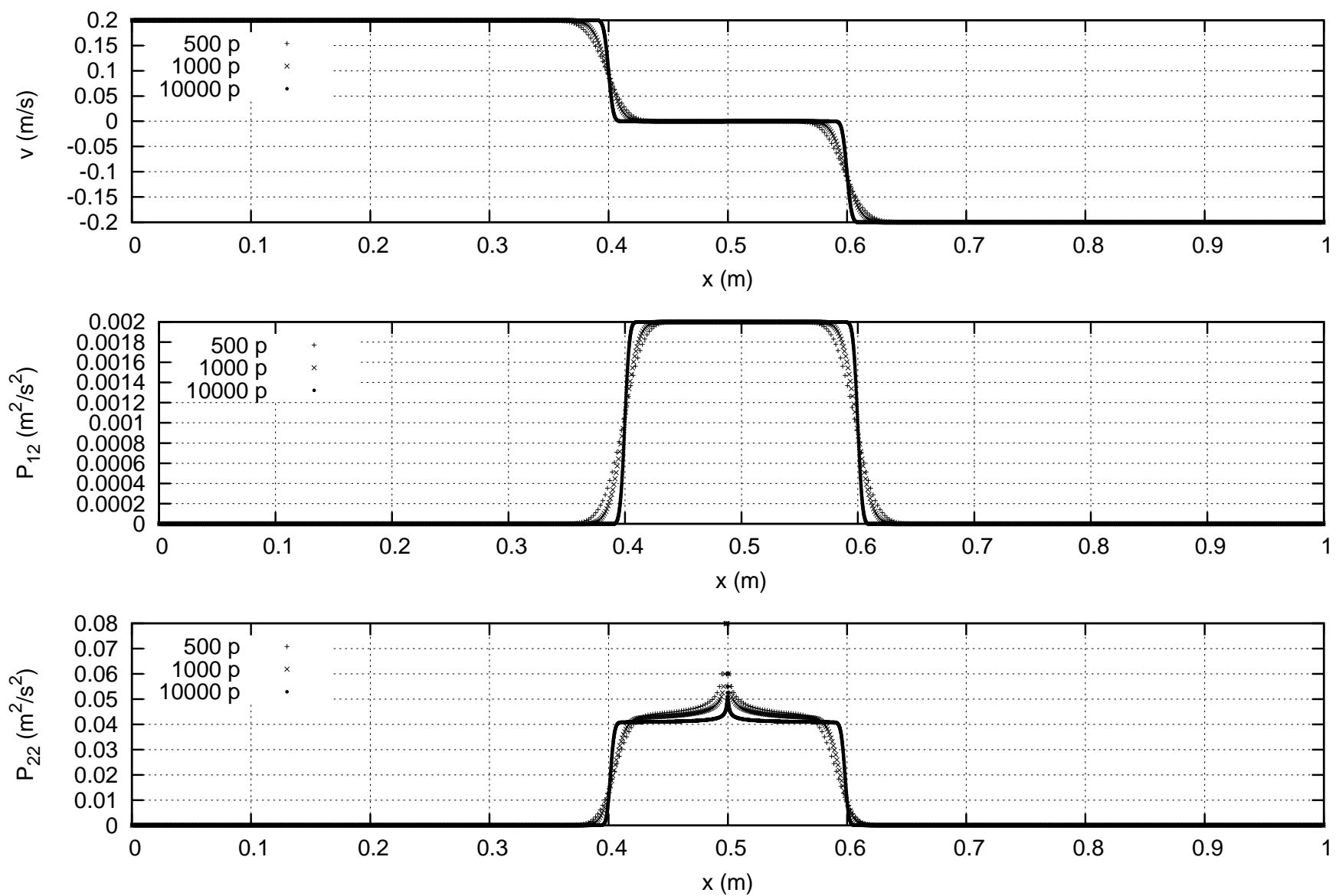

Figure 3: Shear test problem. The initial discontinuity is located at $x=0.5 \mathrm{~m}$. The tangential velocity is $0.2 \mathrm{~m} / \mathrm{s}$ on the left, and $-0.2 \mathrm{~m} / \mathrm{s}$ on the right. The tangential velocity, shear stress $P_{12}$ and stress $P_{22}$ are shown at time instant $10 s$ for 500, 1000 and 10000 grid cells. CFL number is 0.3. 

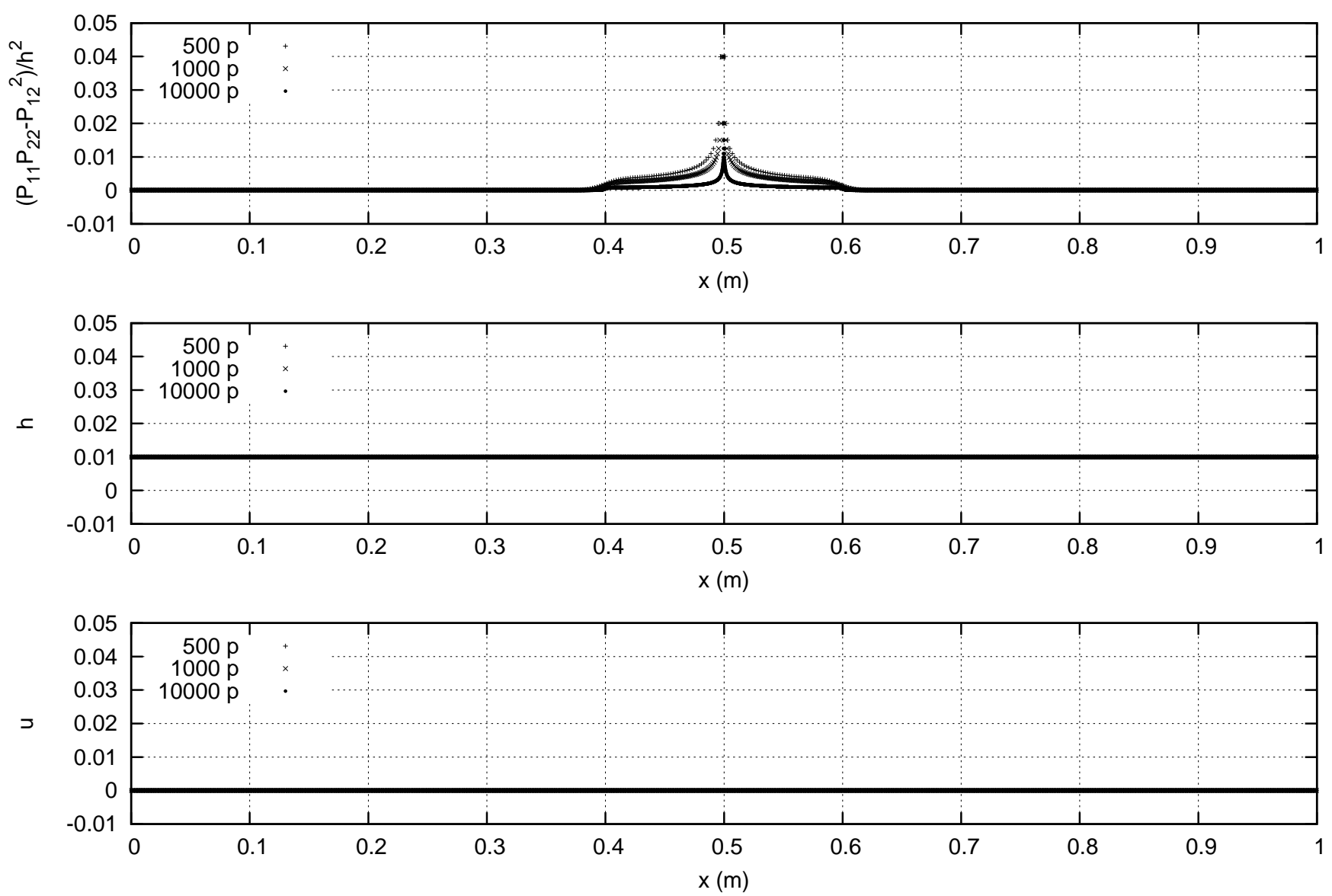

Figure 4: Shear test problem: initially, the discontinuity of the tangential velocity $(0.2 \mathrm{~m} / \mathrm{s}$ on the left and $-0.2 \mathrm{~m} / \mathrm{s}$ on the right ) is at $x=0.5 \mathrm{~m}$. The entropy, depth and normal velocity are shown at time instant $10 \mathrm{~s}$ for 500, 1000 and 10000 grid cells. CFL number is 0.3 . 


\subsection{D dam-break problem}

We solve here the governing equations in the case of vanishing source term. The initial discontinuity is located at $x=0.5 \mathrm{~m}$. The initial normal and tangential velocities are zero everywhere, the components of stress tensor are : $P_{11}=P_{22}=10^{-4} \mathrm{~m}^{2} / \mathrm{s}^{2}, P_{12}=0 \mathrm{~m}^{2} / \mathrm{s}^{2}$, the fluid depth is $0.02 \mathrm{~m}$ at the left and $0.01 \mathrm{~m}$ at the right. The MUSCL extension of Godunov method is used. The results are obtained by using Minmod limiter for the subsystem 1, and van Leer limiter for the subsystem 2. The solution for 100, 1000 and 10000 grid cells is shown in Figure 5. The convergence is clearly visible.
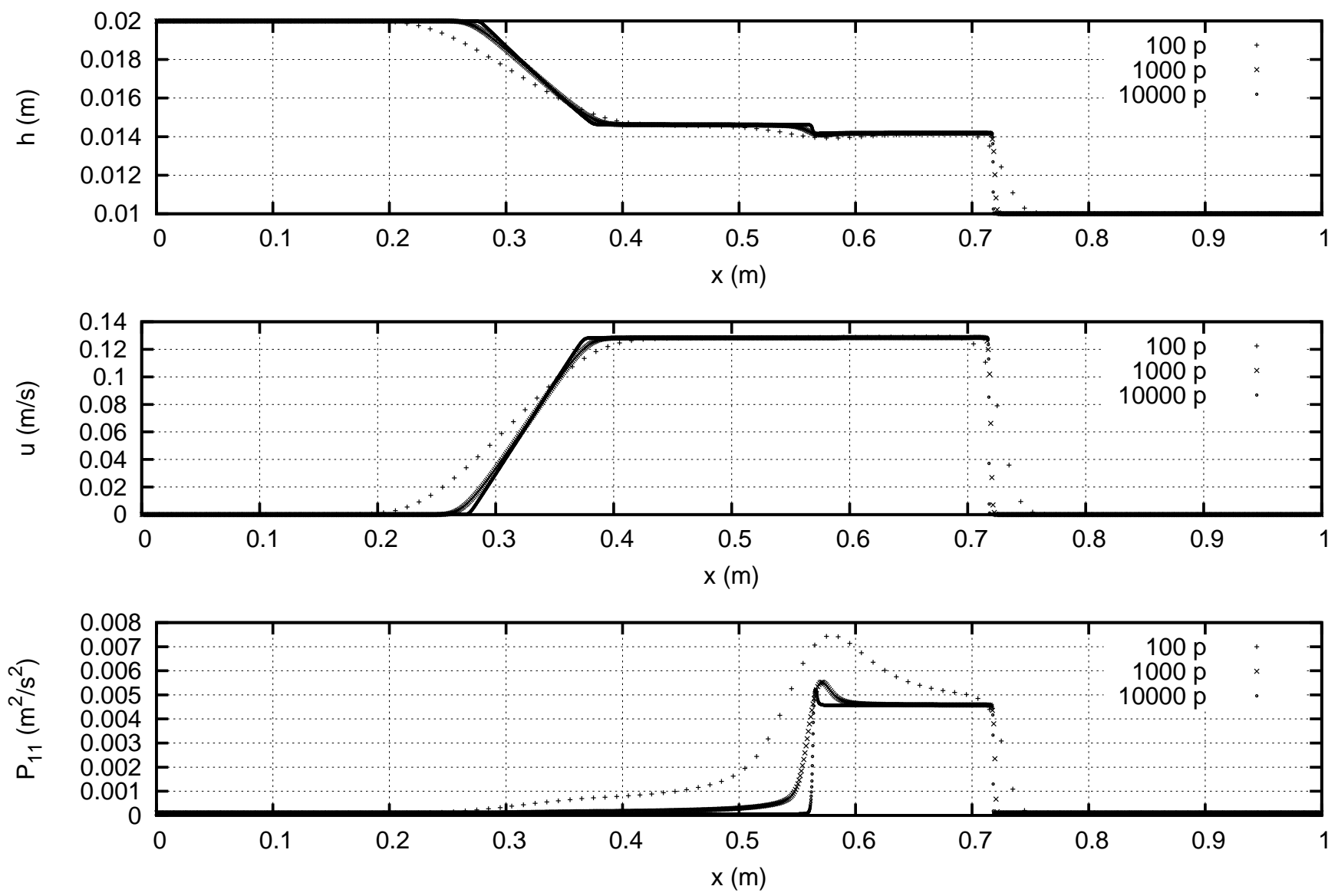

Figure 5: Convergence test in dam-break problem: the initial discontinuity of the depth ( $0.02 \mathrm{~m}$ at the left and $0.01 \mathrm{~m}$ at the right) is located at $x=0.5 \mathrm{~m}$. The fluid depth, normal stress component $P_{11}$ and normal velocity $u$ are shown at time instant $0.5 s$ with 100, 1000, 10000 grid cells. The MUSCL extension of Godunov method is used. CFL number is 0.8. 


\subsection{D roll waves}

We solve here the governing equations with source term. The bottom is inclined either in $x$-direction, or in $y$-direction. Using periodic conditions in the direction of wave propagation, and the rigid wall condition in the transverse direction, we observe the formation of roll waves as in [37, 23]. The initial conditions are taken in the same form as in the above mentioned references. For example, for the flow in $x$-direction, one takes :

$$
\begin{gathered}
h(x, y, 0)=h_{0}\left(1+a \sin \left(\frac{2 \pi x}{L_{x}}\right)\right), u(x, y, 0)=\sqrt{\frac{g h_{0} \tan \theta}{C_{f}}}, v(x, y, 0)=0, \\
P_{11}(x, y, 0)=P_{22}(x, y, 0)=\frac{\varphi h^{2}(x, y, 0)}{2}, P_{12}=0 .
\end{gathered}
$$

Here $\theta=0.05011[\mathrm{rad}]$ is the inclination angle, $C_{f}=0.0036$ is the Chézy coefficient, $h_{0}=7.98 \times$ $10^{-3} \mathrm{~m}, a=0.05, \quad \varphi=22.76 \mathrm{~s}^{-2}, g=9.81 \mathrm{~ms}^{-2}, C_{r}=0.00035, L_{x}=1.3 \mathrm{~m}$. For the flow in $y$-direction the changes in initial conditions are obvious.
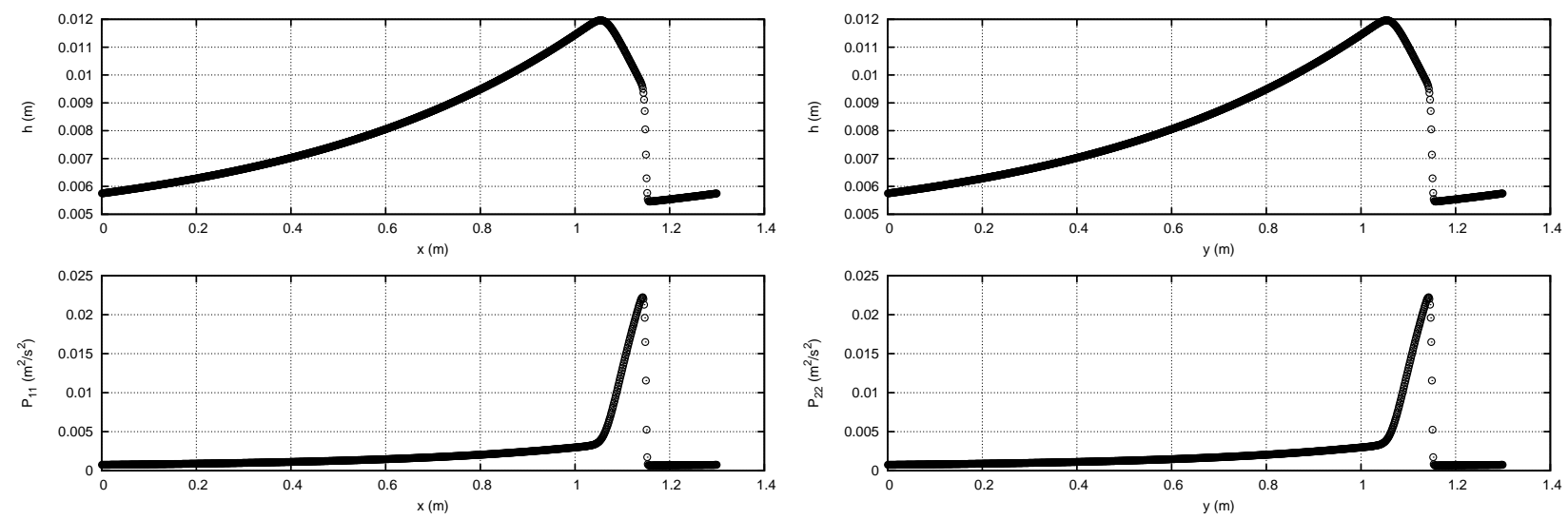

Figure 6: Formation of roll waves : the depth and normal stress are shown at time instant $25 \mathrm{~s}$ for flows both $x$-and $y$-direction (on the left and on the right, respectively). The results are completely symmetric. The MUSCL extension of Godunov method was used with 1000 grid cells for the parameter set mentioned above. CFL number is 0.6.

\subsection{Comparison with a 2D analytical solution}

Here we present analytical solutions to (1). This solution is a generalisation of solutions with linear velocity profile in $x$ an $y$ found by Sedov (1953) and Ovsyannikov (1956) for the Euler equation (for proof, see Appendix B ) :

$$
\left\{\begin{array}{c}
h=\frac{h_{0}}{1+\beta^{2} t^{2}}, \\
\mathbf{U}=\frac{\beta}{1+\beta^{2} t^{2}}\left(\begin{array}{c}
\beta t x+y \\
-x+\beta t y
\end{array}\right), \\
\mathbf{P}=\frac{1}{\left(1+\beta^{2} t^{2}\right)^{2}}\left(\begin{array}{cc}
\lambda+\gamma \beta^{2} t^{2}, & (\lambda-\gamma) \beta t \\
(\lambda-\gamma) \beta t, & \gamma+\lambda \beta^{2} t^{2}
\end{array}\right),
\end{array}\right.
$$


where $h_{0}>0, \beta, \lambda>0, \gamma>0$ are constant. This solution describes the velocity field which is linear in $x$ and $y$. The stress tensor $\mathbf{P}$ is not spherical. We take here $h_{0}=1 \mathrm{~m}, \lambda=0.1 \mathrm{~m}^{2} / \mathrm{s}^{2}$, $\gamma=0.01 \mathrm{~m}^{2} / \mathrm{s}^{2}, \beta=10^{-3} \mathrm{~s}^{-1}, C F L=0.5$. The error was calculated at time instant $10 \mathrm{~s}$ in the following way :

$$
\begin{aligned}
& \operatorname{error}\left(P_{11}\right)=\max _{x, y}\left(\frac{\left|P_{11 \text { numerical }}-P_{11 \text { analytical }}\right|}{\lambda}\right), \\
& \operatorname{error}\left(P_{12}\right)=\max _{x, y}\left(\frac{\left|P_{12 \text { numerical }}-P_{12 \text { analytical }}\right|}{\lambda+\gamma}\right), \\
& \operatorname{error}\left(P_{22}\right)=\max _{x, y}\left(\frac{\left|P_{22 \text { numerical }}-P_{22 \text { analytical }}\right|}{\gamma}\right) .
\end{aligned}
$$

These errors are shown as a function of the grid cell size in the Figure 7 . The calculation region is a square of length $L_{x}=L_{y}=10 \mathrm{~m}$. Different regular Cartesian grids were used $(100 \times 100$, $200 \times 200,400 \times 400,800 \times 800,1600 \times 1600)$ with first order Godunov method. This test shows the convergence to the exact solution. The slope of the convergence lines are the same for all stress components.

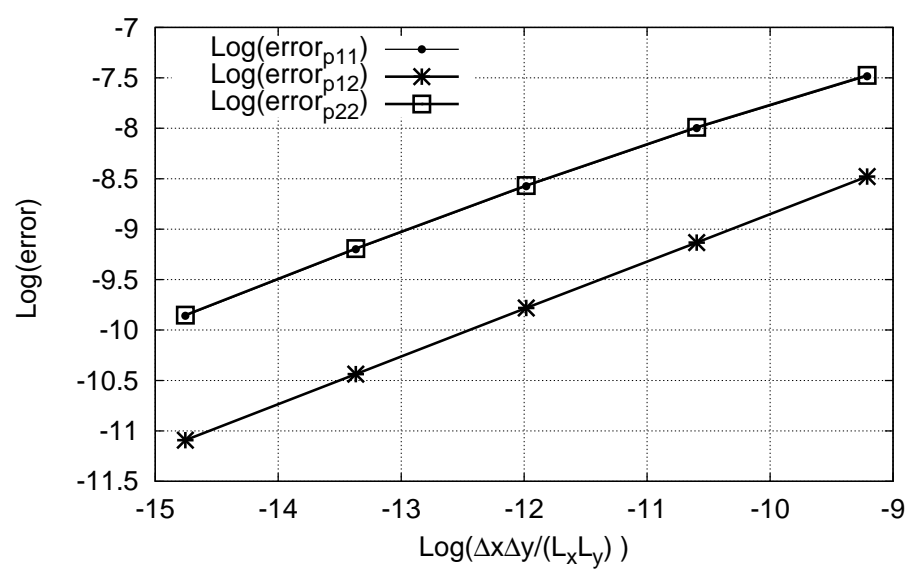

Figure 7: The convergence lines for the stress tensor components as functions of the grid cell size are shown. The number of grid cells was $100 \times 100,200 \times 200,400 \times 400,800 \times 800,1600 \times 1600$. The convergence to the exact solution is clearly visible.

\subsection{D roll waves}

We solve here the $2 \mathrm{D}$ governing equations with source term. The bottom is inclined in $x$-direction. In the direction of wave propagation we use periodic conditions for the vector of unknowns $(\mathbf{U}(0, y, t)=$ $\left.\mathbf{U}\left(L_{x}, y, t\right)\right)$, and in the transverse direction we use the rigid wall conditions $\left(v(x, 0, t)=v\left(0, L_{y}, t\right)=\right.$ $0, P_{12}(x, 0, t)=P_{12}\left(x, L_{y}, t\right)=0, P_{22}(x, 0, t)=P_{22}\left(x, L_{y}, t\right)=0$, and the Neumann conditions for other variables). The initial conditions are taken in the form :

$$
\begin{gathered}
h(x, y, 0)=h_{0}\left(1+a \sin \left(\frac{2 \pi m x}{L_{x}}\right)+a \sin \left(\frac{2 \pi k y}{L_{y}}\right)\right), u(x, y, 0)=\sqrt{\frac{g h_{0} \tan \theta}{C_{f}}}, v(x, y, 0)=0, \\
P_{11}(x, y, 0)=P_{22}(x, y, 0)=\frac{\varphi h^{2}(x, y, 0)}{2}, P_{12}=0 .
\end{gathered}
$$


Here $\theta=0.05011[\mathrm{rad}]$ is the inclination angle, $C_{f}=0.0036$ is the Chézy coefficient, $h_{0}=7.98 \times$ $10^{-3} \mathrm{~m}, \varphi=22.76 \mathrm{~s}^{-2}, g=9.81 \mathrm{~ms}^{-2}, C_{r}=0.00035, L_{x}=1.3 \mathrm{~m}, L_{y}=0.5 \mathrm{~m}$, and $\mathrm{m}$ and $k$ are numbers to be chosen. The set of physical parameters corresponds to that considered in [23] to describe the formation of $1 \mathrm{D}$ roll waves from a uniform flow having the same structure as in Brock's experiments $[10,11,12]$. A necessary condition for the formation of such waves is that the corresponding Froude number is larger than two : $F_{g}=\sqrt{\frac{g h_{0} \tan (\theta)}{C_{f}\left(g h_{0}+3 \varphi h_{0}^{2} / 2\right)}}>2$. For the flow parameters taken above, this value is about 3.7 .

The Figure 8 shows the results of multidimensional computations for a uniform flow perturbed both in $x$ and $y$ direction corresponding to initial data (79) with $m=1$ and $k=1$. The existence of a characteristic transverse wave length of the jump toe perimeter (the line where the gradient of the layer thickness $h$ jumps) is clearly visible. One can count approximately 7 transverse waves.

The transverse structure formation scenario is rather surprising. First, a one-dimensional roll wave is forming, without any transverse structure. This 1D structure is formed in approximately $10 \mathrm{~s}$ and corresponds to a standard 1D experimental profile $[10,11,12]$ (see Figure 9). The transverse structure starts to form in approximately $17 \mathrm{~s}$ and becomes stationary after approximately $35 \mathrm{~s}$. Thus, the whole scenario, from uniform unstable flow with the generalised Froude number $F_{g} \approx$ 3.7 , to $1 \mathrm{D}$ roll waves, and finally to the formation of transverse waves at the jump toe perimeter (formation of 'fingers'), is observed. Such a transverse structure of the bore toe perimeter was, in particular, observed in field experiments [27].

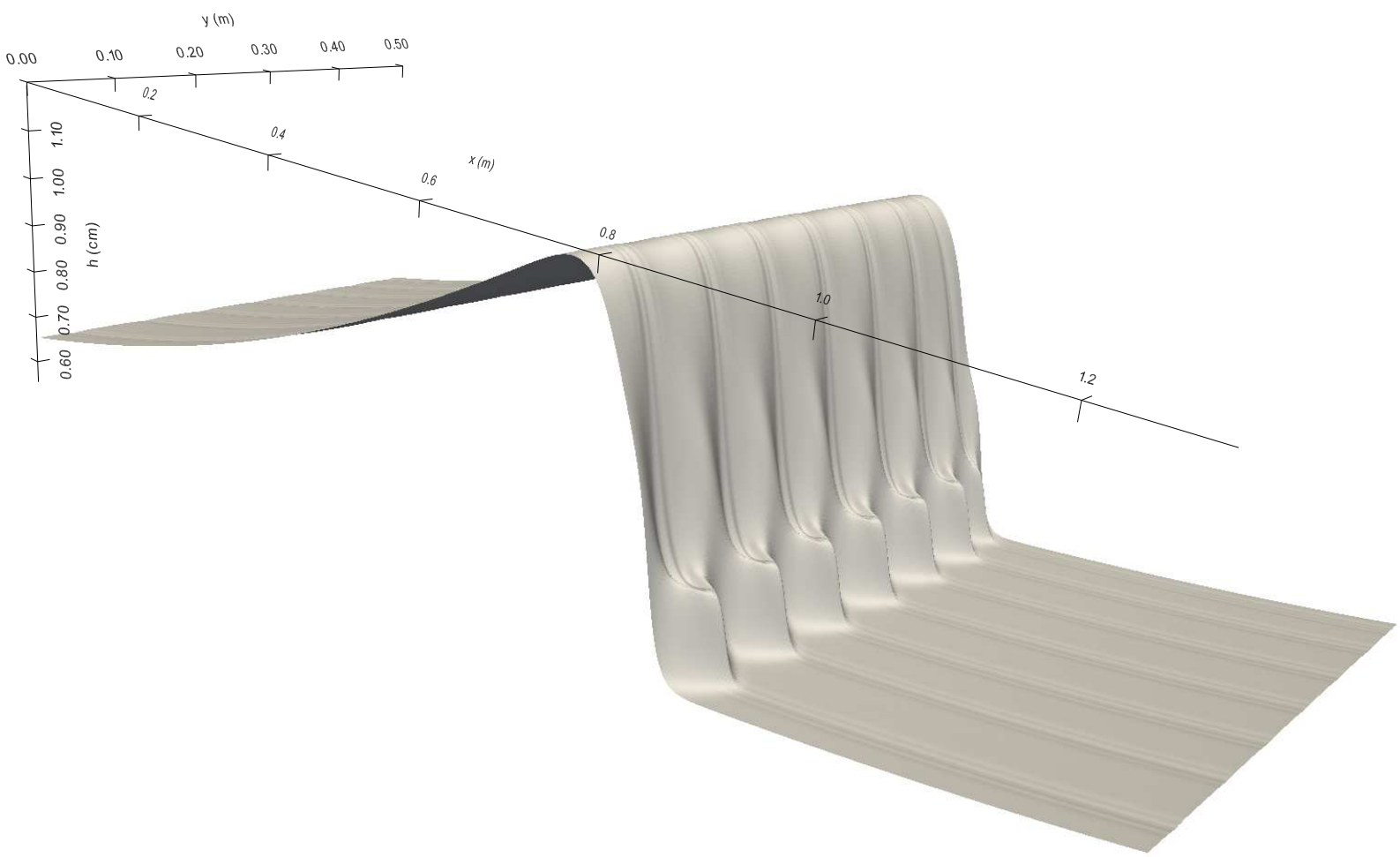

Figure 8: Formation of a transverse structure of the jump toe perimeter consisting of seven waves for the initial data (79) with $m=1$ and $k=1$. The results are obtained for the domain $1.3 \mathrm{~m}$ long and $0.5 \mathrm{~m}$ wide and shown at time instant $39 \mathrm{~s}$. The Godunov method was used with $600 \times 600$ grid cells for the parameter set mentioned above. CFL number is 0.1 .

To understand if the number of transverse waves per unit length is independent on the initial perturbations, we considered also the initial data (79) with $m=1$ and $k=4$. Thus, the initial transverse perturbation contains four waves, and one could expect that the number of transverse 


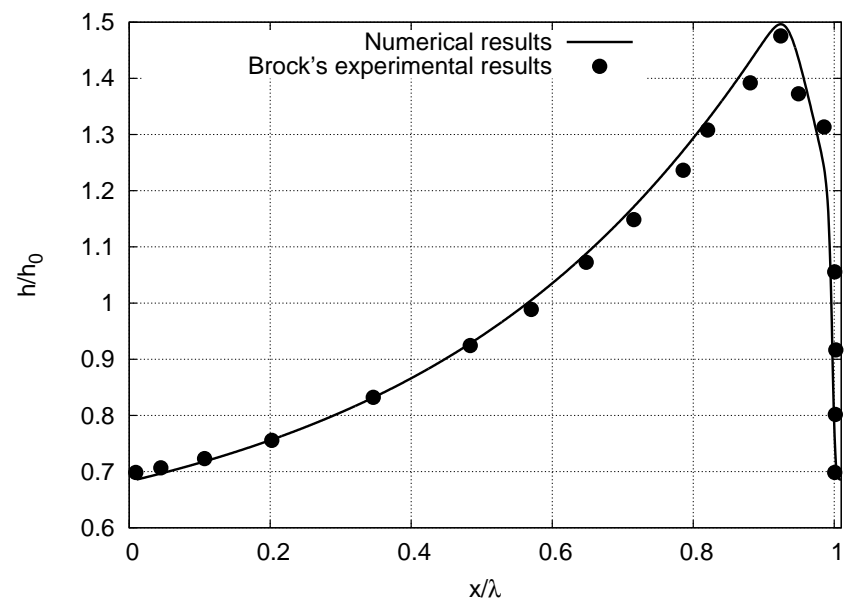

Figure 9: The one-dimensional wave structure is formed in approximately $10 s$ (solid line) and corresponds to the classical experilmental profile (dotes) measured in $[10,11,12]$.

waves formed during the evolution will increase essentially. The result is rather surprising : the number of waves is almost independent on the initial perturbation, only approximately eight waves were formed instead of seven (see Figure 10).

An additional test was also performed to show that the number of transverse waves per unit length is invariant with respect to the domain width. For this, we multiplied the length $L_{y}$ by two $\left(L_{y}=1 \mathrm{~m}\right)$, and took $m=1, k=1$. As a consequence, the number of transverse waves was also multiplied by two (see Figure 11 and 12).

The form of the dissipation term defined by the coefficient $\alpha$ given by (33) is very important. Indeed, this form was chosen to obtain the same dissipation source term $Q$ as in 1D case studied in $[37,38]$. A simplified formula for $\alpha(34)$ will give us only 1D profile, without any transverse structure (see Figure 13). Moreover, the corresponding 1D profile does not correspond to the experimentally observed profiles in $[10,11,12]$ shown in Figure 9.

\section{Conclusion}

We propose a numerical method consisting in 'physical' splitting of the hyperbolic non-conservative equations for shear shallow water flows. Each split subsystem is hyperbolic, contains only one type of waves, and admits the energy and 'entropy' conservation laws. Moreover, such a splitting allows us to naturally define a weak solution to our system which is compatible to the positive definiteness of the Reynolds stress tensor $\mathbf{P}$. The dissipation is introduced in invariant form which also guarantees the positive-definiteness of $\mathbf{P}$. In the limit of one-dimensional flows, the roll waves solutions obtained earlier in $[37,39]$ are recovered.

An interesting feature of the model is the formation of transverse structures at the jump toe perimeter ('fingers') from one-dimensional initial data which are harmonically perturbed in the transverse direction. The number of waves does not depend neither on the amplitude nor on the number of transverse waves in the initial perturbation. Thus, the full transition scenario is observed in the formation of roll waves : from uniform flow to one-dimensional roll waves, and, finally, to 2D transverse 'fingering' of roll wave profiles.

The method can obviously be extended to the general 3D Reynolds averaged models of barotropic flows, because the structure of the governing equations is exactly the same. The case of nonbarotropic turbulent flows demands an additional modelling because the both entropies, physical and 'mathematical', will increase at the shock. Thus it will be necessary to separate such an increase 

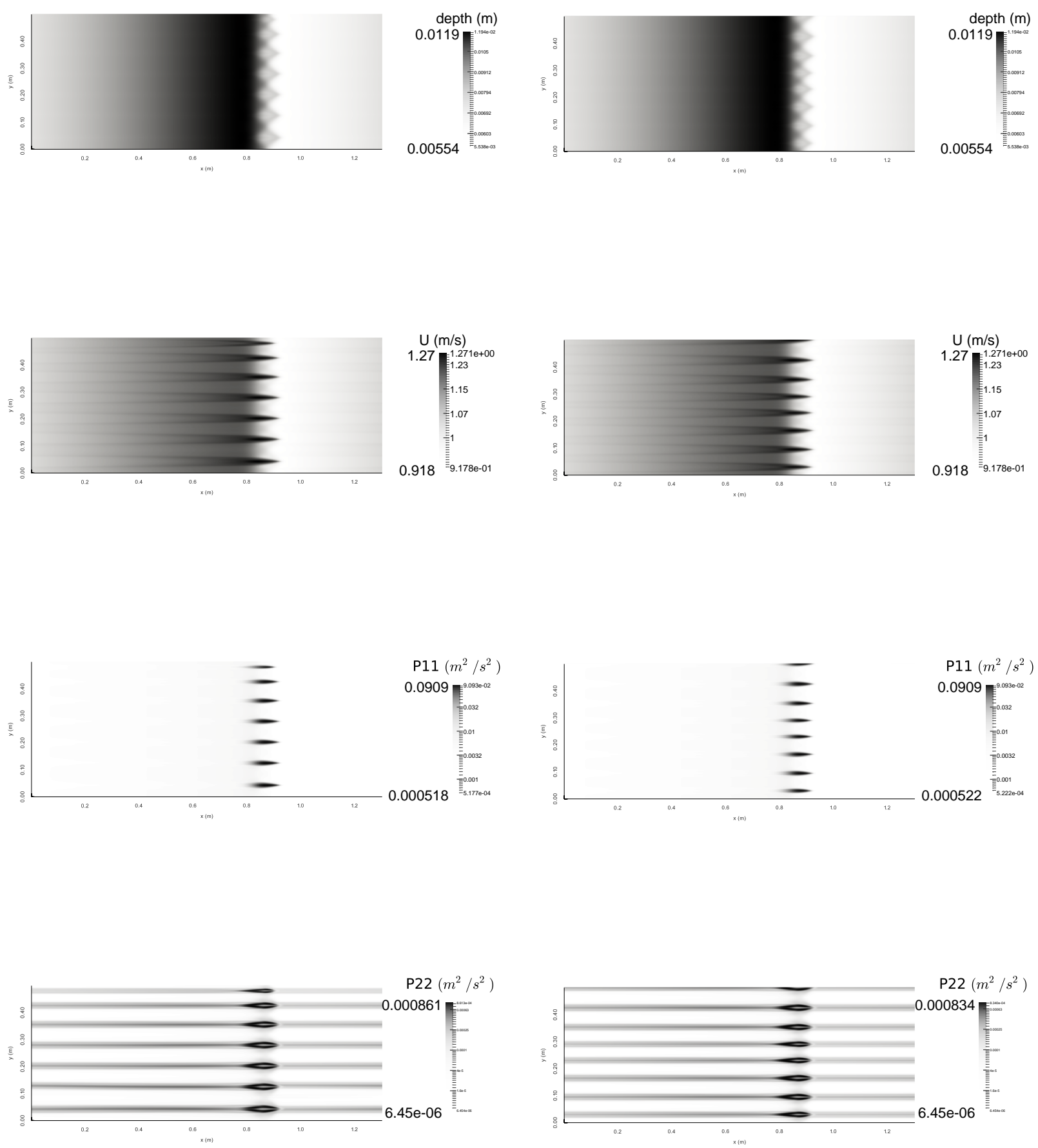

Figure 10: Top view for the schlieren image of the fluid depth, horisontal velocity, and normal stress components $P_{11}$ and $P_{22}$ for the initial perturbation (79) with $k=1, m=1$ (on the left) and $k=1, m=4$ (on the right) at time instant $39 \mathrm{~s}$. The computational domain is $1.3 \mathrm{~m}$ long and $0.5 \mathrm{~m}$ wide. The Godunov method was used, with $600 \times 600$ grid cells for the parameter set mentioned above. CFL number here is 0.1. Seven transverse waves are observed on the left, and almost eight waves on the right. 


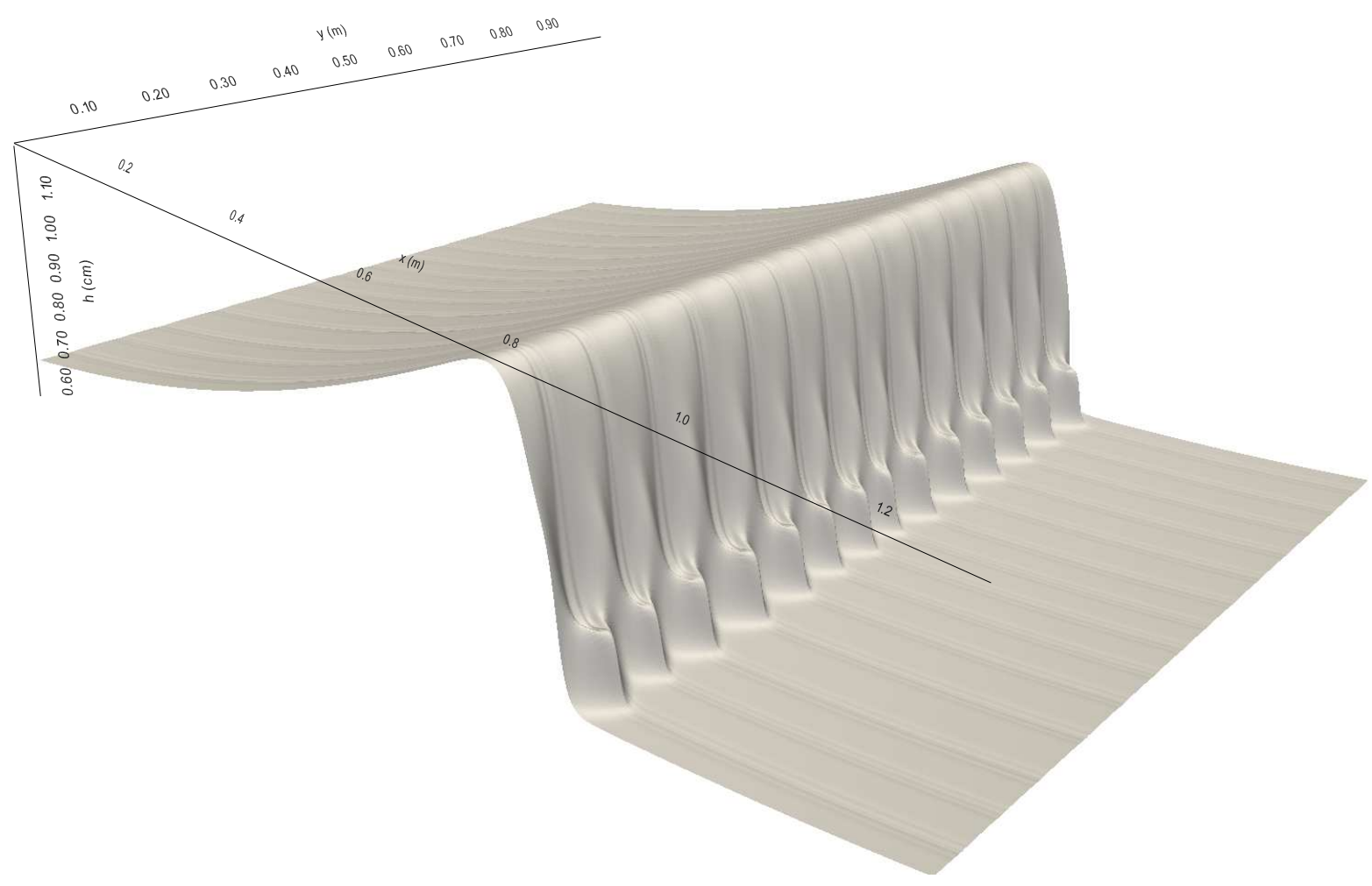

Figure 11: Formation of a transverse structure of the toe perimeter consisting of fourteen waves. The results are obtained for the domain $1.3 \mathrm{~m}$ long and $1 \mathrm{~m}$ wide, and shown at time instant $39 \mathrm{~s}$. The Godunov method was used with $600 \times 1200$ grid cells for the parameter set mentioned above. CFL number is 0.1 .

into two parts : the thermodynamic and turbulent ones. One possibility could be analogous to that introduced in [41] where the whole entropy production was only the turbulent one. This will be the subject of our future work.

Acknowledgement The authors thank Boniface Nkonga for useful discussion. S. L. G. has been partially supported by the ANR project BoND (ANR-13-BS01-0009-01). 

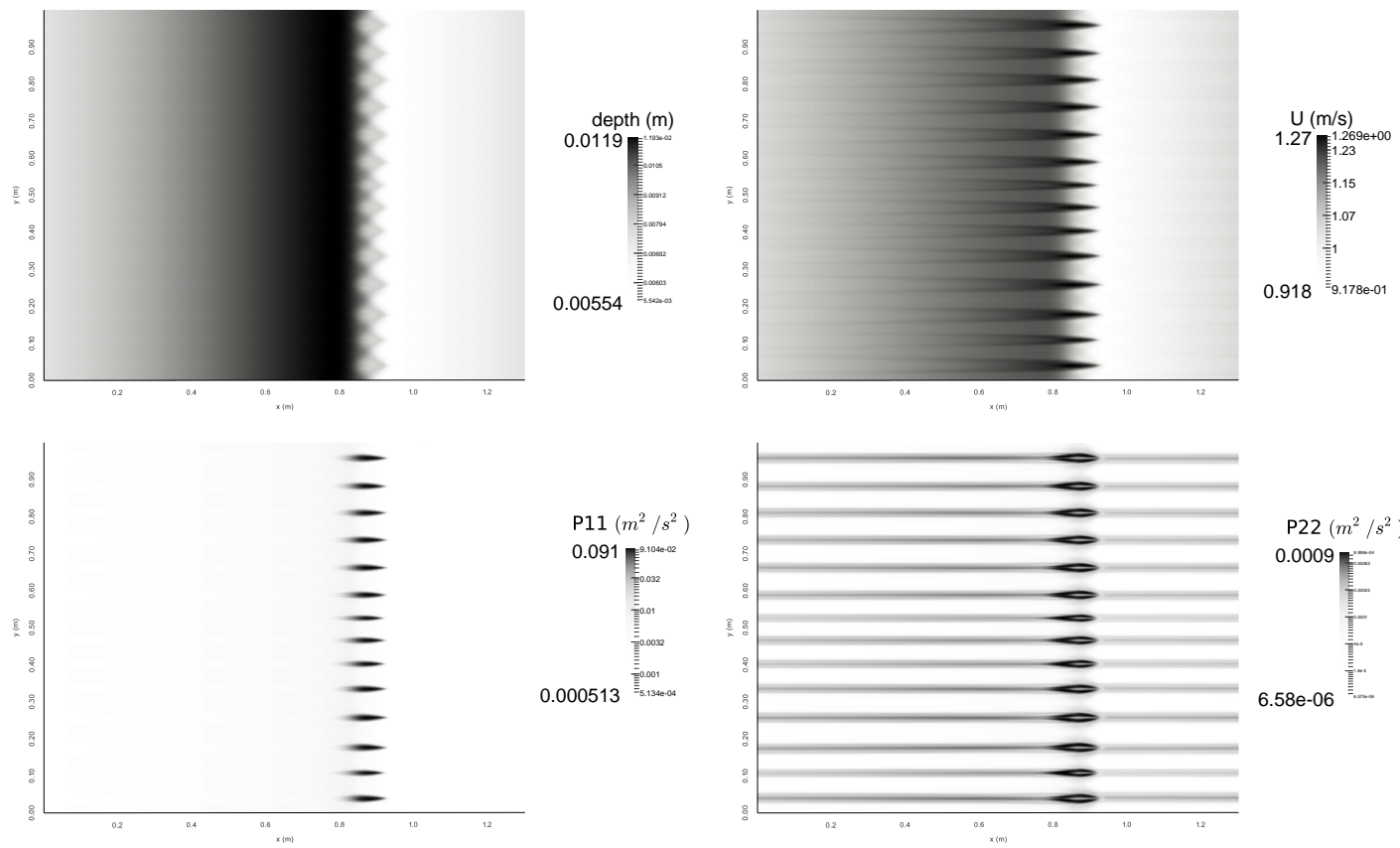

Figure 12: Top view for the schlieren image of the fluid depth $h$, horizontal velocity $u$ and normal stress components $P_{11}$ and $P_{22}$ are shown at the time instant $39 \mathrm{~s}$. The computational domain is $1.3 \mathrm{~m}$ long and $1 \mathrm{~m}$ wide. The Godunov method was used, with $600 \times 1200$ grid cells for the parameter set mentioned above. CFL number here is 0.1. Fourteen transverse waves are observed.

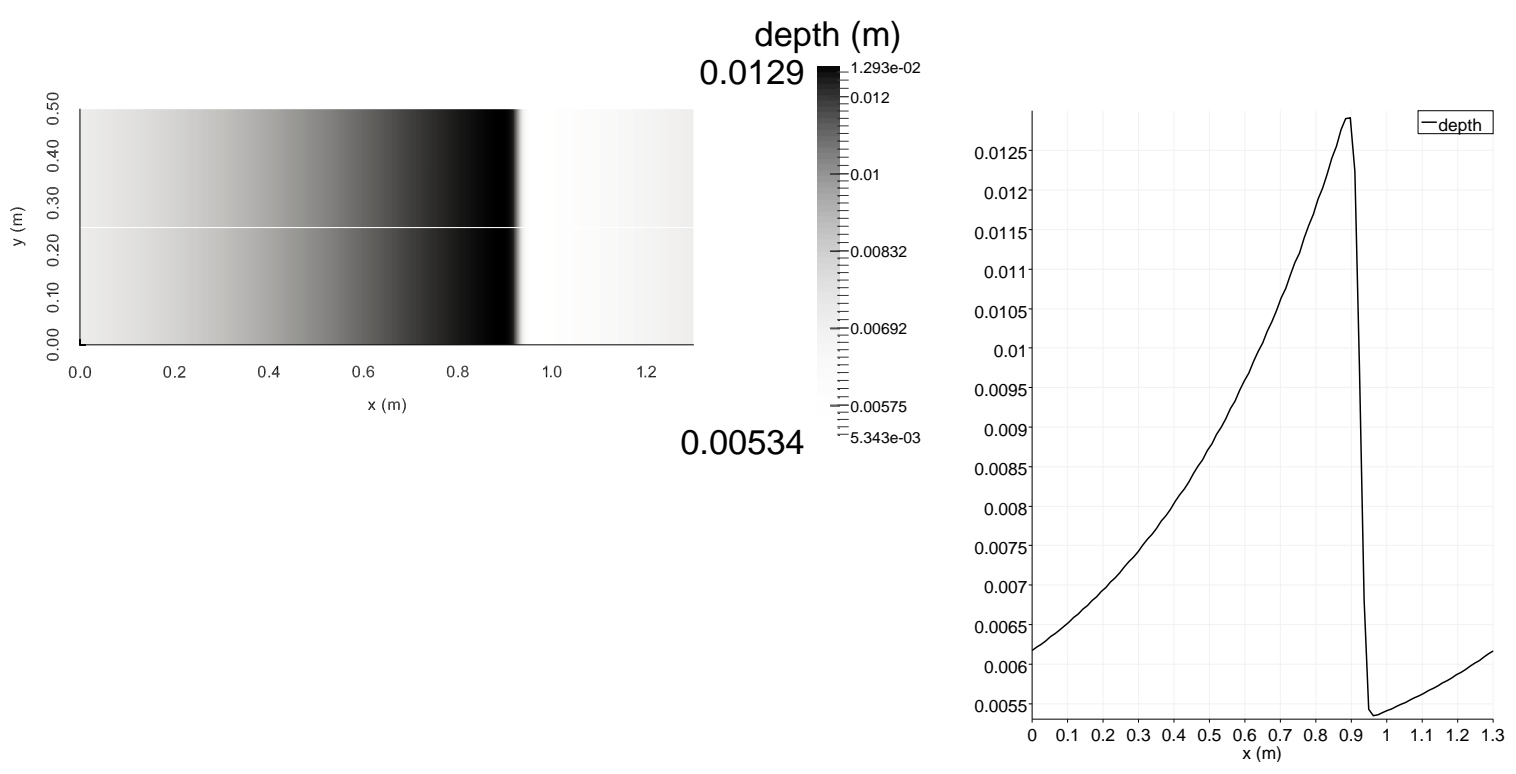

Figure 13: Top view for the schlieren images of the fluid depth (on the left) and depth profile (on the right) with initial data (79) with $m=1$ and $k=4$. The new dissipation law (34) was taken. The result obtained is very surprising : transverse structures do not develop. The second fact, less surprising, is that the one-dimensional profile is not that of Brock's type. 


\section{Appendix A : Conservation laws}

We write down once again the governing equations of shear flows without right hand sides :

$$
\begin{gathered}
h_{t}+u h_{x}+v h_{y}+h u_{x}+h v_{y}=0, \\
u_{t}+u u_{x}+v u_{y}+g h_{x}+\frac{1}{h}\left(h P_{11}\right)_{x}+\frac{1}{h}\left(h P_{12}\right)_{y}=0, \\
v_{t}+u v_{x}+v v_{y}+g h_{y}+\frac{1}{h}\left(h P_{12}\right)_{x}+\frac{1}{h}\left(h P_{22}\right)_{y}=0, \\
P_{11 t}+u P_{11 x}+v P_{11 y}+2 P_{11} u_{x}+2 P_{12} u_{y}=0, \\
P_{12 t}+u P_{12 x}+v P_{12 y}+P_{12}\left(u_{x}+v_{y}\right)+P_{11} v_{x}+P_{22} u_{y}=0, \\
P_{22 t}+u P_{22 x}+v P_{22 y}+2 P_{12} v_{x}+2 P_{22} v_{y}=0 .
\end{gathered}
$$

We are looking for conservation laws admitting by (80) :

$$
\frac{\partial f}{\partial t}+\frac{\partial g_{1}}{\partial x}+\frac{\partial g_{2}}{\partial y}=0
$$

where the unknown functions $f, g_{1}, g_{2}$ depend on $h, \mathbf{u}$ and $\mathbf{P}$. Developing (81) one obtains :

$$
\begin{aligned}
& f_{h} h_{t}+f_{u} u_{t}+f_{v} v_{t}+f_{P_{11}} P_{11 t}+f_{P_{12}} P_{12 t}+f_{P_{22}} P_{22 t}+g_{1 h} h_{x}+g_{1 u} u_{x}+g_{1 v} v_{x}+g_{1 P_{11}} P_{11 x} \\
& +g_{1 P_{12}} P_{12 x}+g_{1 P_{22}} P_{22 x}+g_{2 h} h_{y}+g_{2 u} u_{y}+g_{2 v} v_{y}+g_{2 P_{11}} P_{11 y}+g_{2 P_{12}} P_{12 y}+g_{2 P_{22}} P_{22 y}=0 .
\end{aligned}
$$

Substituting the time derivatives of unknowns from (80) we have :

$$
\begin{aligned}
& f_{h}\left(-h_{x} u-h u_{x}-h_{y} v-h v_{y}\right)+f_{u}\left(-u u_{x}-v u_{y}-g h_{x}-P_{11 x}-P_{12 y}-\frac{P_{11}}{h} h_{x}-\frac{P_{12}}{h} h_{y}\right) \\
& +f_{v}\left(-u v_{x}-v v_{y}-g h_{y}-P_{12 x}-P_{22 y}-\frac{P_{12}}{h} h_{x}-\frac{P_{22}}{h} h_{y}\right)+f_{P_{11}}\left(-u P_{11 x}-v P_{11 y}-2 P_{11} u_{x}-2 P_{12} u_{y}\right) \\
& +f_{P_{12}}\left(-u P_{12 x}-v P_{12 y}-P_{12} u_{x}-P_{22} u_{y}-P_{11} v_{x}-P_{12} v_{y}\right)+f_{P_{22}}\left(-u P_{22 x}-v P_{22 y}-2 P_{12} v_{x}-2 P_{22} v_{y}\right) \\
& +g_{1 h} h_{x}+g_{1 u} u_{x}+g_{1 v} v_{x}+g_{1 P_{11}} P_{11 x}+g_{1 P_{12}} P_{12 x}+g_{1 P_{22}} P_{22 x} \\
& +g_{2 h} h_{y}+g_{2 u} u_{y}+g_{2 v} v_{y}+g_{2 P_{11}} P_{11 y}++g_{2 P_{12}} P_{12 y}+g_{2 P_{22}} P_{22 y}=0 .
\end{aligned}
$$

As the space derivatives of unknowns are independent, and (82) should be satisfied, we obtain the following overdetermined system of equations :

$$
\begin{gathered}
-u f_{h}-g f_{u}-\frac{P_{12}}{h} f_{v}-\frac{P_{11}}{h} f_{u}+g_{1 h}=0, \\
-h f_{h}-u f_{u}-2 P_{11} f_{P_{11}}-P_{12} f_{P_{12}}+g_{1 u}=0, \\
-u f_{v}-2 P_{12} f_{P_{22}}-P_{11} f_{P_{12}}+g_{1 v}=0, \\
-v f_{h}-\frac{P_{12}}{h} f_{u}-g f_{v}-\frac{P_{22}}{h} f_{v}+g_{2 h}=0, \\
-h f_{h}-v f_{v}-2 P_{22} f_{P_{22}}-P_{12} f_{P_{12}}+g_{2 v}=0, \\
-v f_{u}-2 P_{12} f_{P_{11}}-P_{22} f_{P_{12}}+g_{2 u}=0, \\
-f_{u}-u f_{P_{11}}+g_{1 P_{11}}=0, \\
-f_{v}-u f_{P_{12}}+g_{1 P_{12}}=0, \\
-u f_{P_{22}}+g_{1 P_{22}}=0, \\
-f_{u}-v f_{P_{12}}+g_{2 P_{12}}=0, \\
-f_{v}-v f_{P_{22}}+g_{2 P_{22}}=0, \\
-v f_{P_{11}}+g_{2 P_{11}}=0 .
\end{gathered}
$$


A useful remark is that there is no conservation laws with the density $f$ depending only on $\mathbf{P}$. The remark will allow us to neglect in determining $f$ the integration 'constants' depending only on $\mathbf{P}$. Indeed, equations $(83 \mathrm{~g})$ - (831) imply that in this case up to an additive constant $g_{1}=u f, g_{2}=v f$. The equations (83b), (83c), (83e) and (83f) form then a linear with respect to $f$ overdetermined system of equations. The analysis shows that the solution is only trivial.

Eliminating the functions $g_{1}$ and $g_{2}$ from the last 6 equations of (83) we obtain :

$$
\left\{\begin{array}{l}
f_{u P_{11}}=0, \\
f_{u P_{22}}=0, \\
f_{u P_{12}}=0, \\
f_{v P_{11}}=0, \\
f_{v P_{12}}=0, \\
f_{v P_{22}}=0 .
\end{array}\right.
$$

It implies :

$$
f\left(h, u, v, P_{11}, P_{12}, P_{22}\right)=f_{1}(h, u, v)+f_{2}\left(h, P_{11}, P_{12}, P_{22}\right),
$$

where $f_{i}, i=1,2$, are arbitrary functions (in the following, several new arbitrary functions will appear). Eliminating the functions $g_{1}$ and $g_{2}$ from the first 6 equations of (83) we obtain :

$$
\begin{gathered}
h^{2}\left(\frac{f}{h}\right)_{h u}+\left(P_{12} f_{P_{12}}+2 P_{22} f_{P_{22}}\right)_{u}-\left(P_{22} f_{P_{12}}+2 P_{12} f_{P_{11}}\right)_{v}=0, \\
h^{2}\left(\frac{f}{h}\right)_{h v}+\left(P_{12} f_{P_{12}}+2 P_{11} f_{P_{11}}\right)_{v}-\left(P_{11} f_{P_{12}}+2 P_{12} f_{P_{22}}\right)_{u}=0, \\
\left(\frac{P_{12}}{h} f_{u}+\left(g+\frac{P_{22}}{h}\right) f_{v}\right)_{u}-\left(P_{22} f_{P_{12}}+2 P_{12} f_{P_{11}}\right)_{h}=0, \\
\left(\frac{P_{12}}{h} f_{v}+\left(g+\frac{P_{11}}{h}\right)_{u}\right)_{v}-\left(P_{11} f_{P_{12}}+2 P_{12} f_{P_{22}}\right)_{h}=0, \\
\left(\frac{P_{12}}{h} f_{u}+\left(g+\frac{P_{22}}{h}\right) f_{v}\right)_{v}-\left(P_{12} f_{P_{12}}+2 P_{22} f_{P_{22}}\right)_{h}-h f_{h h}=0, \\
\left(\frac{P_{12}}{h} f_{v}+\left(g+\frac{P_{11}}{h}\right) f_{u}\right)_{u}-\left(P_{12} f_{P_{12}}+2 P_{11} f_{P_{11}}\right)_{h}-h f_{h h}=0 .
\end{gathered}
$$

In particular, (84), (85a) and (85b) imply :

$$
\left(\frac{f}{h}\right)_{h u}=0,\left(\frac{f}{h}\right)_{h v}=0 .
$$

Then (84) and (86) imply :

$$
f\left(h, u, v, P_{11}, P_{12}, P_{22}\right)=h c_{1}(u, v)+f_{2}\left(h, P_{11}, P_{12}, P_{22}\right) .
$$

Taking the difference (85c) and (85d), then (85e) and (85f), and differentiating these differences with respect to $u$ and $v$, one can immediately derive that $c_{1}(u, v)$ is quadratic with respect to $u$ and $v$ :

$$
c_{1}(u, v)=k u v+n_{1} u^{2}+n_{2} u+m_{1} v^{2}+m_{2} v
$$


where $k, n_{1}, n_{2}, m_{1}, m_{2}$ are constants. The general form $f$ is then :

$$
f\left(h, u, v, P_{11}, P_{12}, P_{22}\right)=h\left(k u v+n_{1} u^{2}+n_{2} u+m_{1} v^{2}+m_{2} v\right)+f_{2}\left(h, P_{11}, P_{12}, P_{22}\right) .
$$

The next step is thus to determine the function $f_{2}\left(h, P_{11}, P_{12}, P_{22}\right)$ from the overdetermined system obtained by replacing (88) into (85c), (85d), (85e) and (85f):

$$
\begin{gathered}
2 P_{12} n_{1}+\left(g h+P_{22}\right) k-\left(P_{22} f_{2 P_{12}}+2 P_{12} f_{2 P_{11}}\right)_{h}=0, \\
2 P_{12} m_{1}+\left(g h+P_{11}\right) k-\left(P_{11} f_{2 P_{12}}+2 P_{12} f_{2 P_{22}}\right)_{h}=0, \\
P_{12} k+2\left(g h+P_{22}\right) m_{1}-\left(P_{12} f_{2 P_{12}}+2 P_{22} f_{2 P_{22}}\right)_{h}-h f_{2 h h}=0, \\
P_{12} k+2\left(g h+P_{11}\right) n_{1}-\left(P_{12} f_{2 P_{12}}+2 P_{11} f_{2 P_{11}}\right)_{h}-h f_{2 h h}=0 .
\end{gathered}
$$

Taking the difference of $(89 \mathrm{c})$ and $(89 \mathrm{~d})$ and differentiating this difference with respect to $h$, one obtains :

$$
P_{11}\left(f_{2 h h}\right)_{P_{11}}-P_{22}\left(f_{2 h h}\right)_{P_{22}}=g\left(n_{1}-m_{1}\right) .
$$

The general solution of this equation is :

$$
f_{2 h h}=\phi\left(h, P_{12}, x\right)+g\left(n_{1}-m_{1}\right) \ln P_{11}, \text { with } x=P_{11} P_{22} .
$$

Taking now the difference of $(89 \mathrm{a})$ and $(89 \mathrm{~b})$ and differentiating this difference with respect to $h$, one obtains :

$$
\left(P_{11}-P_{22}\right)\left(f_{2 h h}\right)_{P_{12}}+2 P_{12}\left(\left(f_{2 h h}\right)_{P_{22}}-\left(f_{2 h h}\right)_{P_{11}}\right)=0
$$

Substituting (90) into (91) we obtain :

$$
\left(P_{11}^{2}-x\right) \frac{\partial \phi\left(h, P_{12}, x\right)}{\partial P_{12}}+2 P_{12}\left(P_{11}^{2}-x\right) \frac{\partial \phi\left(h, P_{12}, x\right)}{\partial x}-2 P_{12} g\left(m_{1}-n_{1}\right)=0 .
$$

This is a polynomial of degree two in $P_{11}$. Since the identity should be valid for any $P_{11}$, the coefficients of the polynomial vanish. This implies : $\phi_{P_{12}}+2 P_{12} \phi_{x}=0$ and $m_{1}=n_{1}$. Integrating two times in $h$, one obtains the general expression of $f_{2}$ :

$$
f_{2}=\phi_{1}\left(h, P_{11} P_{22}-P_{12}^{2}\right)+h \phi_{2}(\mathbf{P})+\phi_{3}(\mathbf{P}) .
$$

One can always take $\phi_{3}(\mathbf{P})$ vanishing because there is no conservation law with $f$ depending only on $\mathbf{P}$. With the condition $m_{1}=n_{1}$ the difference (89c) -(89d) becomes :

$$
P_{11} \phi_{2 P_{11}}-P_{22} \phi_{2 P_{22}}=\left(P_{11}-P_{22}\right) n_{1} \text {. }
$$

Hence,

$$
\phi_{2}(\mathbf{P})=n_{1}\left(P_{11}+P_{22}\right)+\nu_{1}\left(P_{12}, P_{11} P_{22}\right),
$$

and

$$
f_{2}(h, \mathbf{P})=\phi_{1}\left(h, P_{11} \times P_{22}-P_{12}^{2}\right)+n_{1} h\left(P_{11}+P_{22}\right)+h \nu_{1}\left(P_{12}, P_{11} P_{22}\right) .
$$

We substitute the function $f_{2}(h, \mathbf{P})$ into the equation (89a) and obtain:

$$
\left(g h+P_{22}\right) k-\left(P_{22} \nu_{1 P_{12}}+2 P_{12} P_{22} \nu_{1 x}\right)=0, x=P_{11} P_{22}
$$

It is linear polynomial for $P_{22}$. It identically vanishes if and only if :

$$
k=0, \nu_{1 P_{12}}+2 P_{12} \nu_{1 x}=0
$$

Hence, $\nu_{1}$ is a function of only one argument $\Delta=P_{11} P_{22}-P_{12}^{2}$. One can insert this function into the general expression of $f_{2}$. One has now :

$$
f_{2}(h, \mathbf{P})=\phi_{1}\left(h, P_{11} P_{22}-P_{12}^{2}\right)+n_{1} h\left(P_{11}+P_{22}\right) .
$$


The simplified form of (89) is now :

$$
\begin{gathered}
2 P_{12} n_{1}-\left(P_{22} f_{2 P_{12}}+2 P_{12} f_{2 P_{11}}\right)_{h}=0, \\
2 P_{12} n_{1}-\left(P_{11} f_{2 P_{12}}+2 P_{12} f_{2 P_{22}}\right)_{h}=0, \\
2\left(g h+P_{22}\right) n_{1}-\left(P_{12} f_{2 P_{12}}+2 P_{22} f_{2 P_{22}}\right)_{h}-h f_{2 h h}=0, \\
2\left(g h+P_{11}\right) n_{1}-\left(P_{12} f_{2 P_{12}}+2 P_{11} f_{2 P_{11}}\right)_{h}-h f_{2 h h}=0 .
\end{gathered}
$$

The equations (94a), (94b) are identically satisfied if we replace expression $f_{2}$ given by (93). The equations (94c), (94d) give us the same equation :

$$
2 \Delta\left(\phi_{1 h}\right)_{\Delta}+h\left(\phi_{1 h}\right)_{h}=2 g h n_{1} .
$$

Its solution is :

$$
\phi_{1 h}(h, \Delta)=A\left(\frac{\Delta}{h^{2}}\right)+2 g n_{1} h
$$

with an arbitrary function $A(s)$. Integration with respect to $h$ gives us :

$$
\phi_{1}(h, \Delta)=h B\left(\frac{\Delta}{h^{2}}\right)+n_{1} g h^{2},
$$

where $B(s)$ is an arbitrary function. The final representation of the density function $f$ is :

$$
f(h, \mathbf{u}, \mathbf{P})=h B\left(\frac{P_{11} P_{22}-P_{12}^{2}}{h^{2}}\right)+n_{1}\left(g h^{2}+h\left(P_{11}+P_{22}\right)+h u^{2}+h v^{2}\right)+n_{2} h u+m_{2} h v .
$$

The conservation of mass corresponds to the particular case where $B=$ const.

\section{Appendix B : Analytical solutions}

Here we present analytical solutions to (1). This solution is a generalisation of solutions with linear velocity profile found in Sedov (1953) and Ovsyannikov (1956) for the Euler equations. Thus, we are looking for the solution of the type :

$$
\mathbf{u}=\mathbf{A}(t) \mathbf{x}, \quad h=h(t), \quad \mathbf{P}=\mathbf{P}(t) .
$$

Here $\mathbf{A}(t)$ is a time dependent matrix. System (1) becomes :

$$
\left\{\begin{array}{c}
\dot{h}+h \operatorname{tr}(\mathbf{A})=0, \\
\dot{\mathbf{A}}+\mathbf{A}^{2}=\mathbf{0}, \\
\dot{\mathbf{P}}+\mathbf{A P}+\mathbf{P A}^{T}=\mathbf{0}
\end{array}\right.
$$

Here 'dot' means the time derivative. The solution $\mathbf{A}(t)$ of the second equation of $(96)$ is given in the form :

$$
\mathbf{A}=\mathbf{A}_{0}\left(\mathbf{I}+\mathbf{A}_{0} t\right)^{-1}, \quad \mathbf{A}_{0}=\text { const. }
$$

Since the corresponding matrices commute, $\mathbf{A}$ can also be written as :

$$
\mathbf{A}=\left(\mathbf{I}+\mathbf{A}_{0} t\right)^{-1} \mathbf{A}_{0}
$$

Then $\mathbf{P}(t)$ verifies the equation :

$$
\dot{\mathbf{P}}+\mathbf{A}_{0}\left(\mathbf{I}+\mathbf{A}_{0} t\right)^{-1} \mathbf{P}+\mathbf{P}\left(\mathbf{I}+\mathbf{A}_{0}^{T} t\right)^{-1} \mathbf{A}_{0}^{T}=\mathbf{0}
$$


The solution $\mathbf{P}$ of $(97)$ is :

$$
\mathbf{P}=\left(\mathbf{I}+\mathbf{A}_{0} t\right)^{-1} \mathbf{P}_{0}\left(\mathbf{I}+\mathbf{A}_{0}^{T} t\right)^{-1},
$$

with a constant symmetric positive definite matrix $\mathbf{P}_{0}=\mathbf{P}_{0}^{T}>0$. The solution is well defined for all $t>0$, if the matrix $\mathbf{I}+\mathbf{A}_{0} t$ is invertible for any $t$. This is a case, for example, of antisymmetric matrix $\mathbf{A}_{0}: \mathbf{A}_{0}^{T}=-\mathbf{A}_{0}$. As an example, consider the initial data :

$$
\mathbf{A}_{0}=\left(\begin{array}{cc}
0 & \beta \\
-\beta & 0
\end{array}\right), \mathbf{P}_{0}=\left(\begin{array}{cc}
\lambda & 0 \\
0 & \gamma
\end{array}\right), h=h_{0}
$$

with constants $\beta, \lambda>0, \gamma>0$ and $h_{0}>0$. The solution is :

$$
\mathbf{A}(t)=\frac{\beta}{1+\beta^{2} t^{2}}\left(\begin{array}{cc}
\beta t & 1 \\
-1 & \beta t
\end{array}\right), \mathbf{P}=\frac{1}{\left(1+\beta^{2} t^{2}\right)^{2}}\left(\begin{array}{cc}
\lambda+\gamma \beta^{2} t^{2} & (\lambda-\gamma) \beta t \\
(\lambda-\gamma) \beta t & \gamma+\lambda \beta^{2} t^{2}
\end{array}\right), h=\frac{h_{0}}{1+\beta^{2} t^{2}}
$$

\section{References}

[1] R. Abgrall, S. Karni, Two-layer shallow water systems : a relaxation approach, SIAM J. Sci. Comput.31(3) (2009) 1603-1627.

[2] B. Audebert, F. Coquel, Structural Stability of Shock Solutions of Hyperbolic Systems in Nonconservation Form via Kinetic Relations. In: Benzoni-Gavage S., Serre D. (eds) Hyperbolic Problems: Theory, Numerics, Applications. Springer, Berlin, Heidelberg, 2008.

[3] M. Baer, J. Nunziato, A two-phase mixture theory for the deflagration-to-detonation transition (DDT) in reactive granular materials. Int. J. Multiphase Flows, 12 (1986) 861-889.

[4] P. G. Baines, Topographic effects in stratified flows, Cambridge University Press, Cambridge, 1995.

[5] B. Barros, Conservation laws for one-dimensional shallow water models for one and two-layer flows, Math. Models and Methods in Applied Sciences, 16 (2006) 119-137.

[6] D.--J. Benney, Some properties of long nonlinear waves, Stud. Appl. Math. 52 (1973) 45-50.

[7] C. Berthon, F. Coquel, J.-M. Hérard, M. Uhlmann, An approximate solution of the Riemann problem for a realisable second-moment turbulent closure, Shock Waves, 11 (2002) 245-269.

[8] N. Besse, On the Waterbag Continuum, Arch. Rational Mech. Anal. 199 (2011) 453-491.

[9] F. Bouchut, Nonlinear Stability of Finite Volume Methods for Hyperbolic Conservation Laws and Well-Balanced Schemes for Sources. Birkhäuser, 2004.

[10] R. R. Brock, Development of roll waves in open channels, PhD Thesis, Caltech, 1967.

[11] R. R. Brock, Development of roll-wave trains in open channels, J. Hydraulics Division, 95 (1969) 1401-1428.

[12] R. R. Brock, Periodic permanent roll waves, J. Hydraulics Division, 96 (1970) 2565-2580.

[13] A. Castro, D. Lannes, Fully nonlinear long-wave models in the presence of vorticity, J. Fluid Mech. 759 (2014) 642-675. 
[14] F. Coquel, J.-M. Hérard, K. Saleh, A Positive and Entropy-Satisfying Finite Volume Scheme for the Baer-Nunziato Model, J. Computational Physics 330 (2016) 401-435.

[15] G. Dal Maso, P.-G. LeFloch, F. Murat, Definition and weak stability of a non-conservative product, J. Math. Pures Appli.74(1995) 483-548.

[16] A. N. Dremin, I. A. Karpukhin, Method of determination of shock adiabat of the dispersed substances. Zhurnal Prikladnoi Mekhaniki i Tekhnicheskoi Fiziki,1 (3)(1960) 184-188 (in Russian).

[17] N. Favrie, S.L. Gavrilyuk, Diffuse interface model for compressible fluid- compressible elasticplastic solid interaction, J. Computational Physics, 231 (2012) 2696-2723.

[18] N. Favrie, S. Gavrilyuk, S. Ndanou, A thermodynamically compatible splitting procedure in hyperelasticity, J. Computational Physics, 270 (2014) 300-324.

[19] S. Gavrilyuk, R. Saurel, Estimation of the turbulence energy production across a shock wave, The Journal of Fluid Mechanics, 549 (2006) 131-139.

[20] S. L. Gavrilyuk, H. Gouin, Geometric evolution of the Reynolds stress tensor, Int. J. Engineering Science, 59 (2012) 65-73.

[21] S. Gavrilyuk, Multiphase flow modelling via Hamilton's principle, In the book : F. dell'Isola, S. L. Gavrilyuk (Editors), Variational Models And Methods In Solid And Fluid MechanicsSpringer, 2012.

[22] S. K. Godunov, A difference method for numerical calculation of discontinuous solutions of the equations of hydrodynamics, Matematicheskii Sbornik, 89 (1959) 271-306.

[23] K. A. Ivanova, S. L. Gavrilyuk, B. Nkonga, G. L. Richard, Formation and coarsening of rollwaves in shear shallow water flows down an inclined rectangular channel (submitted)

[24] A. K. Kapila, R. Menikoff, J. B. Bdzil, S. F. Son, D. S. Stewart, Two-phase modeling of deflagration-to-detonation transition in granular materials : reduced equations, Phys. Fluids, 13(10)(2001) 3002-3024.

[25] S. Karni, Multi-component flow calculations by a consistent primitive algorithm, Journal of Computational Physics, 112 (1994) 31-43.

[26] P.--G. LeFloch, Hyperbolic Systems of Conservation Laws. The theory of classical and nonclassical shock waves, Lectures in Mathematics, Birkhäuser, 2002.

[27] X. Leng, H. Chanson, Breaking bore : Physical observation of roller characteristics, Mech. Research Communications, 65(2015) 24-29.

[28] R. J. LeVeque, Numerical methods for conservation laws, Birkhäuser, 1992.

[29] V. Yu. Liapidevskii, V. M. Teshukov, Mathematical models for a long waves propagation in an inhomogeneous fluid, Siberian Branch of the Russian Academy of Sciences, 2000.

[30] K. T. Mandli, Finite volume methods for the multilayer shallow water equations with applications to storm surges: PhD thesis. Washington: Univ. 2011.

[31] B. Mohammadi, O. Pironneau, Analysis of the K-epsilon turbulence model, Research in Applied Mathematics, John Wiley \& Sons, New York, 1994.

[32] P. J. Montgomery, T. G. Moodie, 2001 On the number of conserved quantities for the two-layer shallow water equations, Studies in Applied Mathematics, 106 (2001) 229-259. 
[33] S. Ndanou, N. Favrie, S. Gavrilyuk, Multi-solid and multi-fluid diffuse interface model: applications to dynamic fracture and fragmentation, J. Comput. Phys. 295(2015) 523-555.

[34] L. V. Ovsyannikov, Two-layer shallow water model, Journal of Applied Mechanics and Technical Physics 20 (2) (1979) 127-135.

[35] L. V. Ovsyannikov, A new solution of the hydrodynamic equations, Dokl. AN SSSR 111 (1956) 47-49 (in Russian).

[36] L. V. Ovsyannikov, Lectures on the Fundamentals of Gas Dynamics, Nauka, 1981 (in Russian).

[37] G. L. Richard, S. L. Gavrilyuk, A new model of roll waves: comparison with Brocks experiments, J. Fluid Mechanics, 698 (2012) 374-405.

[38] G. L. Richard, S. L. Gavrilyuk, The classical hydraulic jump in a model of shear shallow-water flows, J. Fluid Mechanics, 725 (2013) 492-521.

[39] G. L. Richard, Elaboration d'un modèle d'écoulements turbulents en faible profondeur: application au ressaut hydraulique et aux trains de rouleaux, PhD thesis, Aix-Marseille Université, 2013 .

[40] R. Saurel, R. Abgrall, A multiphase Godunov method for compressible multifluid and multiphase flows, J. Comput. Physics, 150 (2001) 425-467.

[41] R. Saurel, S. L. Gavrilyuk, F. Renaud, A multiphase model with internal degrees of freedom: application to shock-bubble interaction, Journal of Fluid Mechanics, 495 (2003) 283-321.

[42] R. Saurel, O. Le Metayer, J. Massoni, S. Gavrilyuk, Shock jump relations for multiphase mixtures with stiff mechanical relaxation, Shock Waves, 16 (2007) 209-232.

[43] L. I. Sedov, On the integration of the equations of one-dimensional gas motion, Dokl. AN SSSR 40(1953) 753-755 (in Russian).

[44] V. M. Teshukov, On hyperbolicity of long-wave equations, Soviet Math. Dokl. 32 (1985) 469473.

[45] V. M. Teshukov, On Cauchy problem for long-wave equations. In: Numerical Methods for Free Boundary Problems, ISMN 92, 106 331-338, Birkhäuser, Boston, 1992.

[46] V. Teshukov, G. Russo, A. Chesnokov, Analytical and numerical solutions of the shallow water equations for 2-D rotational flows, Math. Models Methods Appl. Sci. 14 (2004) 1451-1479.

[47] V. M. Teshukov, Gas dynamic analogy for vortex free-boundary flows, Jurnal of Applied Mechanics and Technical Physics, 48 (2007) 303-309.

[48] E. F. Toro, Riemann solvers and numerical methods for fluid dynamics: a practical introduction, Springer, 2009.

[49] O. V. Troshkin, On wave properties of an incompressible turbulent fluid, Physica A 168 (1990) 881-899.

[50] L. Truskinovsky, Kinks versus shocks, R. Fosdick, E. Dunn, H. Slemrod (Eds.), Shock Induced Transitions and Phase Structures in General Media, IMA Vol. Math. Appl. 52, Springer-Verlag, Berlin (1993)

[51] S. B. Pope, Turbulent flows, Cambridge University Press, 2005.

[52] D. Wilcox, Turbulence Modeling for CFD, DCW Industries, 1998. 